\title{
On the formation of the counter-rotating vortex pair in transverse jets
}

\author{
By L. CORTELEZZI $\dagger$ AND A. R. KARAGOZIAN \\ Department of Mechanical and Aerospace Engineering, \\ University of California, Los Angeles, CA 90095-1597, USA
}

(Received 14 May 2000 and in revised form 24 May 2001)

Among the important physical phenomena associated with the jet in crossflow is the formation and evolution of vortical structures in the flow field, in particular the counter-rotating vortex pair (CVP) associated with the jet cross-section. The present computational study focuses on the mechanisms for the dynamical generation and evolution of these vortical structures. Transient numerical simulations of the flow field are performed using three-dimensional vortex elements. Vortex ring rollup, interactions, tilting, and folding are observed in the near field, consistent with the ideas described in the experimental work of Kelso, Lim \& Perry (1996), for example. The time-averaged effect of these jet shear layer vortices, even over a single period of their evolution, is seen to result in initiation of the CVP. Further insight into the topology of the flow field, the formation of wake vortices, the entrainment of crossflow, and the effect of upstream boundary layer thickness is also provided in this study.

\section{Introduction}

The jet in crossflow or transverse jet has been studied over many years because of its applications in a wide variety of technological systems, including fuel or dilution air injection in gas turbine engines and thrust vector control. Among the important physical phenomena associated with the jet in crossflow is the formation of vortical structures which are observed to strongly influence jet behaviour. Some of the earliest studies of this flow field (Kamotani \& Greber 1972; Fearn \& Weston 1974) identified the formation of a counter-rotating vortex pair (CVP) flow structure which was observed to dominate the cross-section of the jet, particularly in the far field (e.g. beyond 5-10 diameters downstream of injection). These time-averaged vortical structures are suggested to be associated with enhanced overall mixing efficiency for the transverse jet as compared with the free jet or mixing layer (Broadwell \& Breidenthal 1984). A schematic diagram of the global or mean flow features of the single jet in crossflow is provided in figure 1.

While the global features of the transverse jet can be understood and predicted in terms of the dynamics of the CVP, the generation and evolution of these vortical structures is not completely understood. Broadwell \& Breidenthal (1984) suggest that the CVP arises from the impulse associated with the jet and thus is a global feature of the far field, in which the CVP is convected downstream by the crossflow. Karagozian $(1986 a, b)$ was able to use vorticity associated with the jet impulse as

$\dagger$ Currently at the Department of Mechanical Engineering, McGill University, Montreal, Quebec, Canada; e-mail crtlz@ametista.mecheng.mcgill.ca 


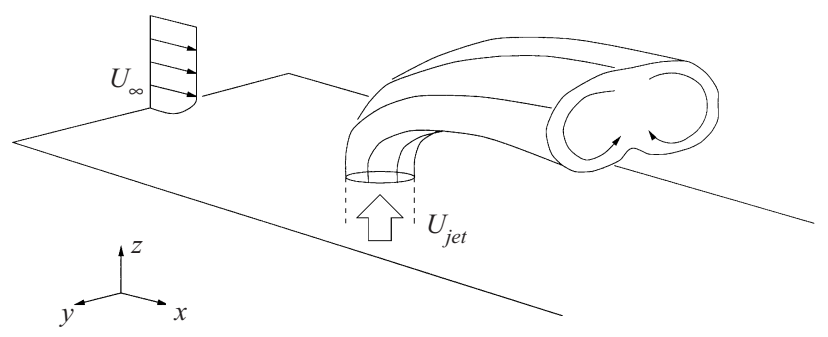

FIGURE 1. Flow geometry for a jet of velocity $U_{\text {jet }}$ in a crossflow of free-stream velocity $U_{\infty}$.

well as that generated by deflection of the crossflow about the jet in the nearfield to predict average vortex pair strength, jet trajectories, and reaction lengths. More detailed experimental studies in the near field have suggested that the CVP is formed by the vortex sheet emanating from the jet nozzle or pipe (Moussa, Trischka \& Eskinazi 1977; Andreopoulos \& Rodi 1984; Fric 1990; Kelso, Lim \& Perry 1996). The recent experiments of Kelso et al. (1996) in both water and air suggest that periodic vortex ring rollup from the nozzle occurs for the jet in crossflow (similar to that which occurs in the free jet), yet superposed on this process is a re-orientation of this shear layer vorticity imposed by the crossflow, which leads to a folding of the cylindrical vortex sheet. The superposition of these two mechanisms results in the interpretation of the evolution of the jet shear layer vortex rings as shown in figure 2(a), taken from the paper by Kelso et al. (1996), where there is a tilting of the upstream portion of the ring oriented with the mean curvature of the jet, and a tilting and folding of the downstream portion of the ring aligned with the direction of the jet. The re-orientation of the shear layer that is seen to lead to this tilting is shown schematically in figure $2(b)$. It is this tilting and folding which is thought by a number of groups (Moussa et al. 1977; Andreopoulos \& Rodi 1984; Kelso et al. 1996) to contribute to the circulation of the CVP. The experiments of Kelso et al. (1996) suggest, for example, that 'the shear layer of the jet folds and rolls up very near to the pipe exit, leading to or contributing to the formation of the CVP'. It should be noted as well that two recent experimental investigations (Smith \& Mungal 1998; Kuzo 1995) suggest that, instantaneously, the CVP can be either symmetric or asymmetric in shape under specific circumstances, and that end views of the jet in the far field can reveal axisymmetric as well as sinusoidal motion of the CVP.

The jet in crossflow is also thought to be influenced by the presence of other vortex systems in the flow field. Horseshoe vortices forming upstream of the jet can influence and be coupled with periodic vortices which form in the wake of the jet (Krothapalli, Lourenco \& Buchlin 1990, Kelso \& Smits 1995). The structure of wake vortices (wall vortices and upright vortices forming in the wake of the jet) have been studied experimentally (Moussa et al. 1977; Fric \& Roshko 1994; Smith \& Mungal 1998). The experiments of Fric \& Roshko (1994) suggest that the vorticity in the wake region originates from the injection wall boundary layer, where the boundary layer fluid wraps around the jet, separating on its lee side and acting to form the upright vortices.

The simulation of a transverse jet constitutes a challenge for any numerical scheme. This flow field is in general three-dimensional, unsteady, and, for many applications, compressible. The formation of the jet, i.e. the initial transient, is of crucial importance in studying the evolution of the jet. Past numerical simulations of transverse jets 
(a)

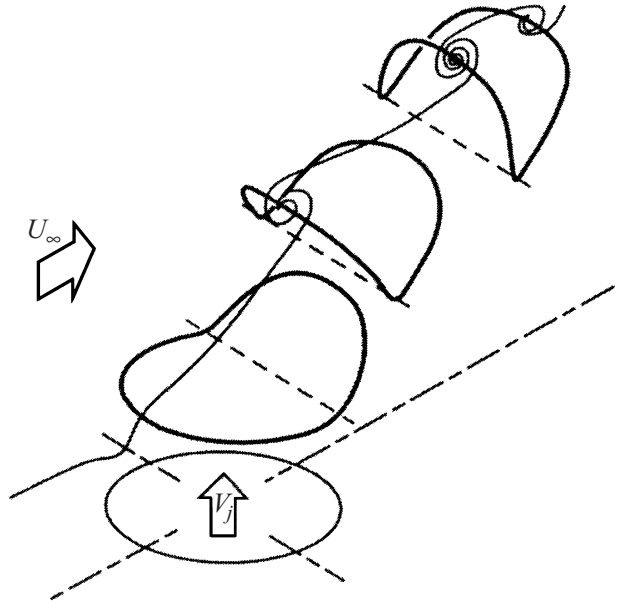

(b)

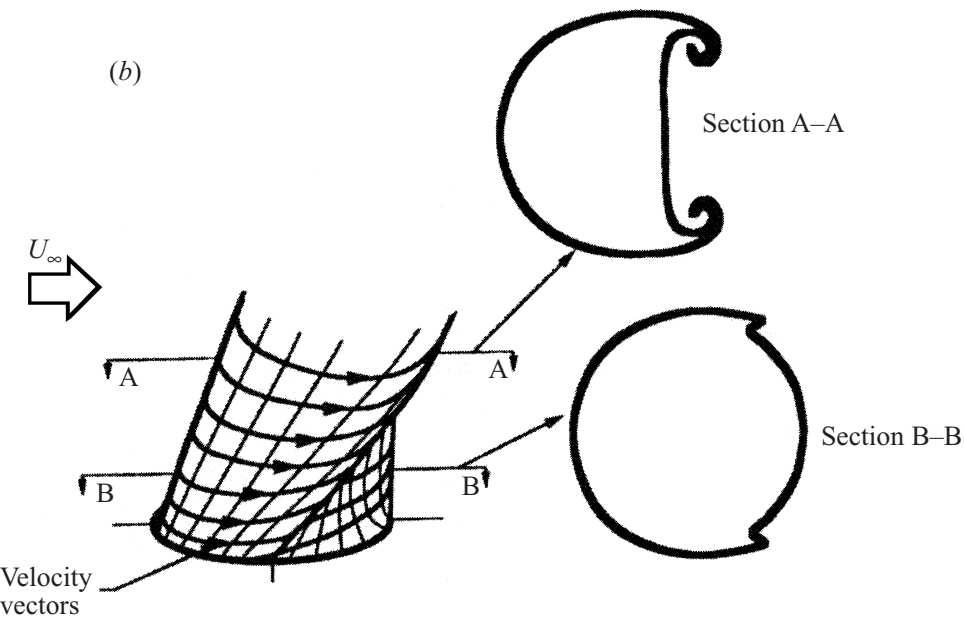

FiguRE 2. The interpretation of Kelso et al. (1996) for shear layer evolution in the transverse jet: (a) isometric view of the jet shear layer vortex rings, showing how they tilt and fold as they convect downstream, and $(b)$ schematic diagram of the reorientation of the shear layer vorticity, leading to the folding of the cylindrical vortex sheet. These correspond to figures 10 and $9(b)$, respectively, in Kelso et al. (1996).

(e.g. Uenishi, Rogers \& Northam 1989) have incorporated turbulence models such as $k-\epsilon$, while the three-dimensional Navier-Stokes calculations by Rudman (1994) successfully capture many details of the laminar evolution of the flow. Another more recent computational study of the jet in crossflow is that of Yuan \& Street (1998) and Yuan, Street \& Ferziger (1999), in which a large-eddy simulation (LES) of the incompressible turbulent flow field is performed. Computed features of the transient jet evolution as well as turbulent stresses are seen to compare well with experimental data, although direct quantitative comparisons of detailed flow features are only possible when the computational input conditions (jet-to-crossflow velocity ratio, jet Reynolds number) are made to be different from those stated in the experiments, possibly due to different upstream pipe flow conditions. These differences could also arise due to the fact that, in many experimental studies, the jet flow characteristics are measured with the crossflow turned off, with the assumption that the jet flow at 
the exit plane and within the nozzle is not strongly influenced by the crossflow. This assumption may not be entirely accurate, as will be discussed further in $\S 2$.

Vortex methods are particularly useful in simulating unbounded, incompressible, unsteady flows where the fluid containing vorticity occupies only a small fraction of the total fluid volume. Vortex methods permit accurate, gridless simulation of the evolution of the vorticity field. The velocity field can then be reconstructed from the vorticity field. In the past 25 years, many vortex schemes have been proposed for the simulation of two- and three-dimensional flows (e.g. Leonard 1985; Sarpkaya 1989; Gharakhani \& Ghoniem 1997; Cottet \& Koumoutsakos 2000). Several features are common among these schemes: the vorticity field is discretized, the position of the vortex elements is identified by Lagrangian coordinates, and the evolution of the vortex elements is computed. The velocity field associated with a vortex element is often referred to as the 'induced' velocity field. Vortex elements move in the velocity field induced by themselves, by other vortex elements, and by the background velocity field. The nonlinear process of mutual induction constitutes the foundation of vortex methods.

Vortex methods have been used to simulate some aspects of the evolution of jets. A number of investigators have studied the evolution of a cylindrical vortex sheet in space (Ashurst 1983; Martin \& Meiburg 1991, 1992; Dahm, Frieler \& Tryggvason 1992). The characteristics of a bifurcating jet at high Reynolds numbers have been studied by Parekh et al. (1988), while more recently, Nitsche \& Krasny (1994) simulated the formation of a vortex ring at the edge of a circular tube, with excellent corroboration with experimental observations. An inviscid three-dimensional vortex sheet model was used to study the near-field flow in the vicinity of the orifice of a transverse jet by Coelho \& Hunt (1989). The vortex sheet model included a distribution of sinks as well as vortices, used to simulate the complex distortion of the vorticity field as well as entrainment just above the injection wall. This complex distortion will be explored in the present study as well.

The present study focuses on the characterization of the near-field penetration and evolution of the transverse jet. Vortex methods are used to simulate and analyse the generation and evolution of the vorticity field. We confirm the mechanism proposed by Kelso et al. (1996) and others describing the formation of the CVP. Furthermore, we suggest an approach which reconciles results obtained from instantaneous measurements or flow visualizations and ensemble-averaged measurements. Finally, we characterize the near-field entrainment of the crossflow into the jet and suggest mechanisms for the initiation of the CVP as well as the initiation of downstream columnar vortices as observed experimentally.

\section{Mathematical formulation}

The flow field induced by a jet in crossflow is in general three-dimensional and unsteady. We model this flow using three-dimensional vortex filaments to characterize the generation and evolution of the vorticity field. A series of assumptions is made in order to isolate the mechanisms for the production of vorticity in this problem. First we consider the injectant and crossflow to be incompressible, with the same density $\rho$. We neglect the coupling between the jet and the wall boundary layer upstream of the jet. The influence of the crossflow on the jet flow at the orifice is computed here (via postprocessing of data), but the effect of the crossflow on the flow deep within the jet nozzle is not represented; this effect is under investigation in a separate study. The present problem is made dimensionless using the radius of the jet, $D / 2$, as a 
characteristic length, and the characteristic time, $D / 2 U_{\infty}$, where $D$ is the diameter of the jet and $U_{\infty}$ the mean crossflow velocity.

The assumption that the crossflow does not affect the flow deep within the pipe allows us to build an underlying potential flow in the problem by superimposing a mean jet flow perpendicular to a mean crossflow. The mean crossflow velocity $U_{\infty}$ is in the $x$-direction over the jet plane $x, y$ in the upper half-space $z \geqslant 0$ (see the geometry indicated in figure 1). The crossflow is prescribed to have a laminar boundary layer of constant thickness $\delta$, with a cubic profile (i.e. $U=U_{\infty}\left[\frac{3}{2} z / \delta-\frac{1}{2}(z / \delta)^{3}\right]$ ) for $0<z \leqslant \delta$ upstream of the jet. The mean jet flow, i.e. the flow through a semi-infinite pipe that discharges into the half-space $z \geqslant 0$, is represented by a semi-infinite, cylindrical vortex sheet of diameter $D$ and circulation $\gamma$ per unit length. The axis of the cylinder coincides with the negative $z$-axis (see figure 1). An analytic solution that relates the bulk or average jet velocity $U_{\text {jet }}$ to the circulation per unit length $\gamma$ of a semi-infinite, cylindrical vortex sheet was derived by Parekh, Leonard \& Reynolds (1988). This is used as a preliminary estimate in the present model, yet, as will be discussed below, the influence of the evolving vorticity in the flow field (above the injection wall) affects the specific value of $U_{j e t}$ present in the vicinity of the exit plane.

We model the evolution of the jet shear layer by introducing a vortex filament near the jet exit at each time step $\mathrm{d} t$. The vortex filaments used in this simulation are three-dimensional vortex rings of finite core size. The $i$ th vortex filament is represented by a set of $N_{i}$ nodes interpolated by a cubic spline. The velocity field induced by all vortex filaments at a point $\boldsymbol{r}$ in space may be computed using the following modified Biot-Savart integral:

$$
\boldsymbol{u}(\boldsymbol{r})=-\sum_{i=1}^{N_{f}} \frac{\Gamma_{i}}{4 \pi} \int_{C_{i}} \frac{\left[\boldsymbol{r}-\boldsymbol{r}_{i}(\eta, t)\right]}{\left[\boldsymbol{r}-\left.\boldsymbol{r}_{i}(\eta, t)\right|^{2}+\alpha \sigma_{i}^{2}\right]^{3 / 2}} \times \frac{\partial \boldsymbol{r}_{i}}{\partial \eta} \mathrm{d} \eta,
$$

where $N_{f}$ is the total number of vortex filaments and $\Gamma_{i} \equiv \gamma^{2} \mathrm{~d} t / 2, \sigma_{i}$ and $C_{i}$ are the circulation, the core radius, and the contour of the $i$ th vortex filament, respectively, situated at position $\boldsymbol{r}_{i}$. The parameter $\alpha$ controls the vorticity distribution within the vortex core. For the computation shown here we set $\alpha=0.413$ to impose a Gaussian distribution within the core, whose size $\sigma_{i}$ changes in time due to vortex stretching (see below). The computation is advanced in time via a second-order predictor/corrector scheme. Since the vortex core size is not considered to be affected by viscous diffusion in the present computations, the simulation may be regarded to be one examining the essentially inviscid evolution of vorticity in the flow field, e.g. as done for three-dimensional shear layers by Ashurst \& Meiburg (1988).

The circulation, the initial position, and the number of nodes of the vortex filaments are crucial to an accurate simulation of the transverse jet. Recently, experimental work by Kelso et al. (1996) showed that the vorticity lines near the jet exit are tilted with respect to the jet plane. The circulation assigned to the filaments at a given time step may thus be deduced from the vorticity convected through the orifice. The initial number of nodes is chosen so that the cubic spline that interpolates them accurately approximates a circle of radius $D / 2$. For the computations shown here, the initial number of nodes is 24; further refinement in the computation with respect to the initial number of nodes yields virtually identical results. The initial distance of the filament from the jet plane is determined by the velocity of the fluid in the pipe. The initial tilt of the filament with respect to the jet plane is chosen so as to minimize the radial velocity of the fluid at the jet exit. This tilt models the result of the interaction between the jet and the crossflow at injection (see figure $2 b$ ), although 
there are clearly physical mechanisms associated with this asymmetry which are not represented.

It is important to note that a vortex filament does not stretch uniformly as it is affected by the crossflow. Filaments actually stretch differently depending on their position along the vortex sheet. As will be seen, and as originally proposed by Kelso et al. (1996), the downstream portion of a filament whose plane becomes aligned with the direction of the jet stretches the most because this part of the filament wraps around the upstream part of the secondary vortex ring formed previously. Stretching and deformation of the filaments is resolved in the present computation using the cubic spline interpolation algorithm, which permits accurate and efficient addition and redistribution of nodes as stretching and deformation occurs in order to maintain an accurate simulation. Hence a typical computation might yield as many as 200 nodes per vortex filament by the time the filament has travelled about four jet radii downstream of injection. The spline scheme also permits computation of the velocity induced at a point, accounting for the contribution due to the curvature of the filaments. The tradeoff in using a spline scheme, however, is that the velocity induced by the spline filament must be calculated by quadrature, which adds to the computational expense.

Maintaining an appropriate level of overlap between adjacent vortex filaments is also important for an accurate simulation. Here the initial core size is assigned to the filament based on the thickness of the boundary layer in the pipe. The time step is chosen so as to guarantee good overlap between adjacent filaments near the jet exit. Vortex stretching tends to reduce the level of overlapping, however; stretching tends to separate the filaments along the jet axis and to increase their length, thus decreasing the size of the filament cores. The change in core radius of the $i$ th filament, $\sigma_{i}$, due to stretching, may be tracked by the following equation:

$$
\frac{\mathrm{d}}{\mathrm{d} t}\left(\sigma_{i}^{2} L_{i}\right)=0
$$

where $L_{i}$ is the instantaneous length of the $i$ th filament. This approach, which maintains a constant vortex filament volume, has been used in simulations of threedimensional shear layers (Ashurst \& Meiburg 1988), for example. Although the detrimental effects of stretching could be balanced using a computationally expensive redistribution of the vorticity field, this is not implemented in the present strategy. The level of overlap for the duration of the near-field computations performed here is found to be satisfactory because computations performed with larger core sizes yielded virtually identical results to those shown in the present study.

Appropriate boundary conditions for the simulation of the jet in crossflow are difficult to implement because of topological complexity arising from the presence of the pipe. There were several alternative boundary conditions examined in the present simulations. First, image vortex filaments were imposed in order to represent the jet injection wall boundary. Second, panel methods were used to represent the injection wall and the jet orifice. Third, image vortex filaments and panel methods were used jointly. Panels were placed within the jet orifice in order to cancel the effect of the images and produce the specified jet velocity. The result of the refinements implemented by panel methods alone or in conjunction with the image vortex filaments were less accurate in the vicinity of the jet orifice because of the poor behaviour of the panels near the jet rim. Simulations with only image vortex filaments produced the most reasonable results, although the nonlinear interaction of the vortex system with the incoming shear layer vorticity right at the injection plane is not precisely 
represented. Yet in all simulations, irrespective of the type of boundary conditions imposed, the same jet flow field is generated in time for $z>D / 8$, attesting to the robustness of the vortex system generated for the jet in crossflow. Results shown in the present study are for the case where image vortex filaments are used to represent the boundary conditions.

The nonlinear, time-dependent evolution of and interactions within the vortex system in fact create a different evolving centerline jet velocity at and in the vicinity of the injection plane than is predicted by the relation of Parekh et al. (1988). The initial transient in the flow, as the jet is started, causes an increase in the jet velocity in time until sustained vortex rollup and tilting occurs, after which the jet centreline velocity essentially remains constant in time, only oscillating slightly in magnitude at the frequency of rollup of the vortex rings. For example, many of the baseline cases shown in the following sections consider a transverse jet with a non-dimensional boundary layer thickness of $\delta / D=0.5$, and with an initial distribution of circulation for a vortex sheet which would correspond to a non-dimensionalized maximum jet velocity of 2 according to the relation of Parekh et al. (1988). But with this input condition, after the evolution of the transverse jet has taken place, the vorticity in the flow field has a chance to affect the jet exit plane conditions such that the velocity in the vicinity of the jet exit plane increases. The baseline case actually produces a peak jet velocity (at the centreline) of $U_{\text {jet }}=10.8$, or a mean non-dimensional jet velocity (which also corresponds approximately to the velocity at $(x, y, z)=(D / 8,0, D / 8))$ of $U_{\text {jet }}=5.4$. The present computation thus requires a postprocessing of the vorticity field to compute the actual mean or peak jet velocity.

\section{Time-resolved near-field evolution of the jet in crossflow}

In this section we present results for the near-field evolution of a single circular jet in crossflow. Emphasis in the investigation is placed on cases where the ratio between the mean jet and free-stream velocities lies between 2 and 11. These three-dimensional vortex simulations thus may be regarded as representing the low-speed flow dynamics seen in the experimental studies of Kelso et al. (1996) and Lim et al. (1998) for the jet in crossflow.

Figure 3 shows the side view of the evolution of a jet in a crossflow when the mean jet-to-crossflow velocity ratio (or non-dimensional jet velocity) is $U_{\text {jet }} / U_{\infty}=5.4$ and the boundary layer thickness is $\delta / D=0.5$. As noted in $\S 2$, the nonlinear interaction of the vortex system with the jet flow creates a higher mean jet velocity than that initiated at the beginning of the computation (with the velocity computed according to Parekh et al. 1988), so that the actual mean jet velocity near the exit plane is computed by postprocessing the vorticity field. The evolution of the jet is represented by plotting the position of all of the nodes of the vortex filaments at a given time, projected onto the $(x, z)$ plane at $y=0$. At non-dimensional time $t=1.5$ only a large vortical structure generated by the initial rollup of the separating shear layer dominates the flow. The starting vortex ring is tilted with respect to the jet plane and the rollup of the vortex sheet is tighter on the upstream side, as indicated in figure $3(a)$. At time $t=2.0$, the size of the starting vortex ring, the tilt angle, and the asymmetry between upstream and downstream rollup have increased. The tilt angle and the asymmetry are responsible for the deformation of the cylindrical vortex sheet that connects the starting vortex ring to the jet exit (figure $3 b$ ). The cylindrical vortex sheet stretches on the upstream side while it starts to fold on the downstream side.

Folding is the first indication of the formation of the CVP, as observed by Kelso et 

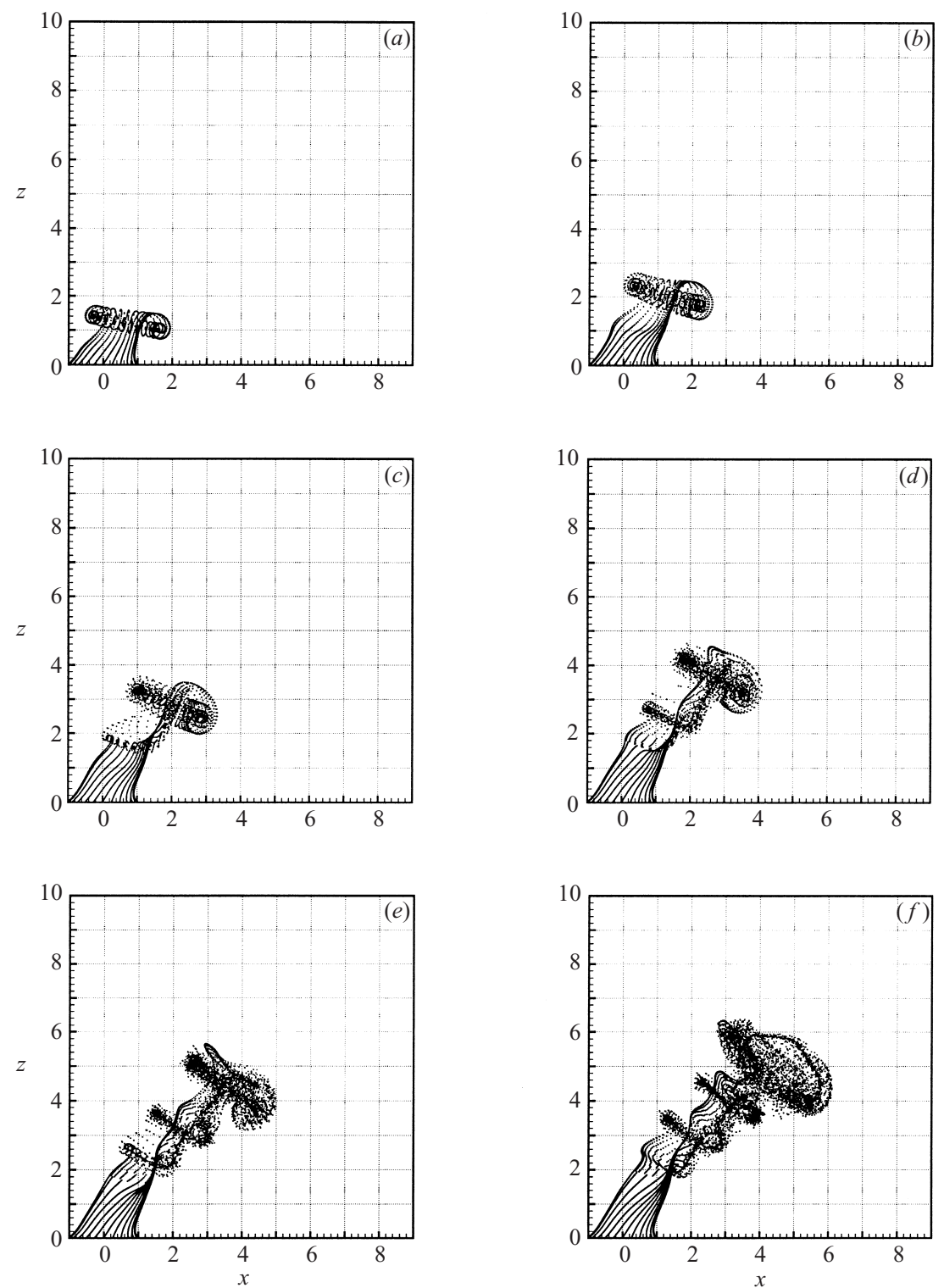

FigURE 3. Side view of the evolution of the transverse jet: $U_{\text {jet }} / U_{\infty}=5.4, \delta / D=0.5$, and $(a) t=1.5$, (b) 2.0, (c) 2.5, (d) 3.0, (e) 3.5, and ( f ) 4.0. Vortex filaments with initially 24 nodes and core size $\sigma^{2}=0.1$ are introduced in the flow at each time step $\mathrm{d} t=0.01$.

al. (1996). At time $t=2.5$, this folding has triggered a roll-up of the cylindrical vortex sheet starting the formation of a secondary vortex ring (figure $3 c$ ). At time $t=3.0$, the formation of the secondary vortex ring is completed. The secondary vortex ring is bent nearly at a right angle with respect to its initial orientation (figure $3 d$ ). Thus, as suggested by Kelso et al. (1996), this secondary vortex ring has folded into two parts: ' ... the upstream part whose plane tilts with the mean curvature of the jet, and the downstream part whose plane becomes aligned with the direction of the jet, and so the sides of the downstream part contribute to the circulation of the CVP'. Figure 3(d) also shows that the side of the secondary vortex ring aligned with the direction of 
the jet begins to wind around the downstream part of the (new) starting vortex ring. As a consequence, the asymmetry of the secondary vortex ring is responsible for the deformation of the cylindrical vortex sheet that connects the secondary vortex ring to the jet exit, in turn generating a new fold. The process of folding and the consequent generation of a new secondary vortex ring repeats itself. At time $t=3.5$, a new secondary vortex ring is formed and a new fold is recognizable. The side of the new secondary vortex ring aligned with the direction of the jet rolls up, in a sort of a spiral, around the side aligned with the direction of the jet of the previous secondary vortex ring, as indicated in figure 3(e). Note that the winding process stretches the vortex filaments, increasing the vorticity in the direction of the jet. Finally, at time $t=4.0$, a new secondary vortex ring is forming (see figure $3 f$ ), which repeats the process of rollup, folding, and interaction with prior and new vortex ring structures. There appears to be a precise period of formation of the secondary vortex rings. For the conditions shown in figure 3 the non-dimensional period $T$ is about 0.68 , where $T$ is made non-dimensional by the jet radius and the crossflow velocity. At flow times beyond 4.0, the interaction between the starting vortex ring and the first and second secondary vortex rings generates a complex topology of the vortex filaments, indicating a breakdown of the vortical structures.

Figures 4 and 5 show the side view of the evolution of a jet in a crossflow when the velocity ratio, or non-dimensional mean jet velocity, is $U_{j e t} / U_{\infty}=2.5$ and 10.8 , respectively, and the boundary layer thickness is $\delta / D=0.5$. A comparison of figures 3-5 shows that the mechanisms of vortex rollup and bending described for the case $U_{\text {jet }} / U_{\infty}=5.4$ apply as well to the case $U_{\text {jet }} / U_{\infty}=2.5$ and 10.8. Folding and generation of secondary vortex rings alternates as in the previous case, although the temporal and spatial scales are different. The characteristic periods of formation of the secondary vortex rings in figures 4 and 5 are approximately $T=1.0$ and 0.38 , respectively. These observations suggest that the frequency of rollup increases with increasing jet velocity, at least in the regime explored here.

Figures 3-5 show good agreement between vortex filament simulations and the experimental observations pertaining to vortex rollup in the transverse jet as presented by Kelso et al. (1996). On the other hand, figures 3-5 do not provide strong evidence for the sustained existence of the global CVP nor for its formation. No further information is provided by the instantaneous velocity vector field in the $(x, y)$ and $(y, z)$-planes. Figures $6(a)$ and $6(b)$ show the velocity vector fields in the $(y, z)$-crossplane at the downstream locations $x=2$ and 3, while figures $6(c)$ and $6(d)$ present the velocity vector field looking down on the jet, in the $(x, y)$-plane parallel to the jet exit plane at the locations $z=2$ and 3, above the injection wall, for the case where $U_{\text {jet }} / U_{\infty}=5.4$ at non-dimensional time $t=4.05$. The dotted line in figure $6(c, d)$ shows the location of the jet exit at $z=0$. Figure $7(a-d)$ shows corresponding vector fields at non-dimensional time $t=4.35$. It is not clear from these instantaneous images that the streamwise vorticity field $\omega_{x}$ at a given location will produce the characteristic kidney-shaped CVP observed in a large number of experimental studies (e.g. Kamotani \& Greber 1972; Moussa et al. 1977; Broadwell \& Breidenthal 1984; Andreopoulos \& Rodi 1984; Fric 1990; Kelso et al. 1996). Only figures 6(c-d) and $7(c-d)$ indicate some structure in the $\omega_{z}$ component of the vorticity field which contributes, as we will show in $\S 4$, to the formation of the CVP. In $\S 4$ we will provide evidence that the formation and existence of the CVP is at least in part an outcome of the averaging process inherent to most experimental studies, consistent with the experimental observations of Smith \& Mungal (1998).

One may also explore the effect of the upstream wall boundary layer thickness 

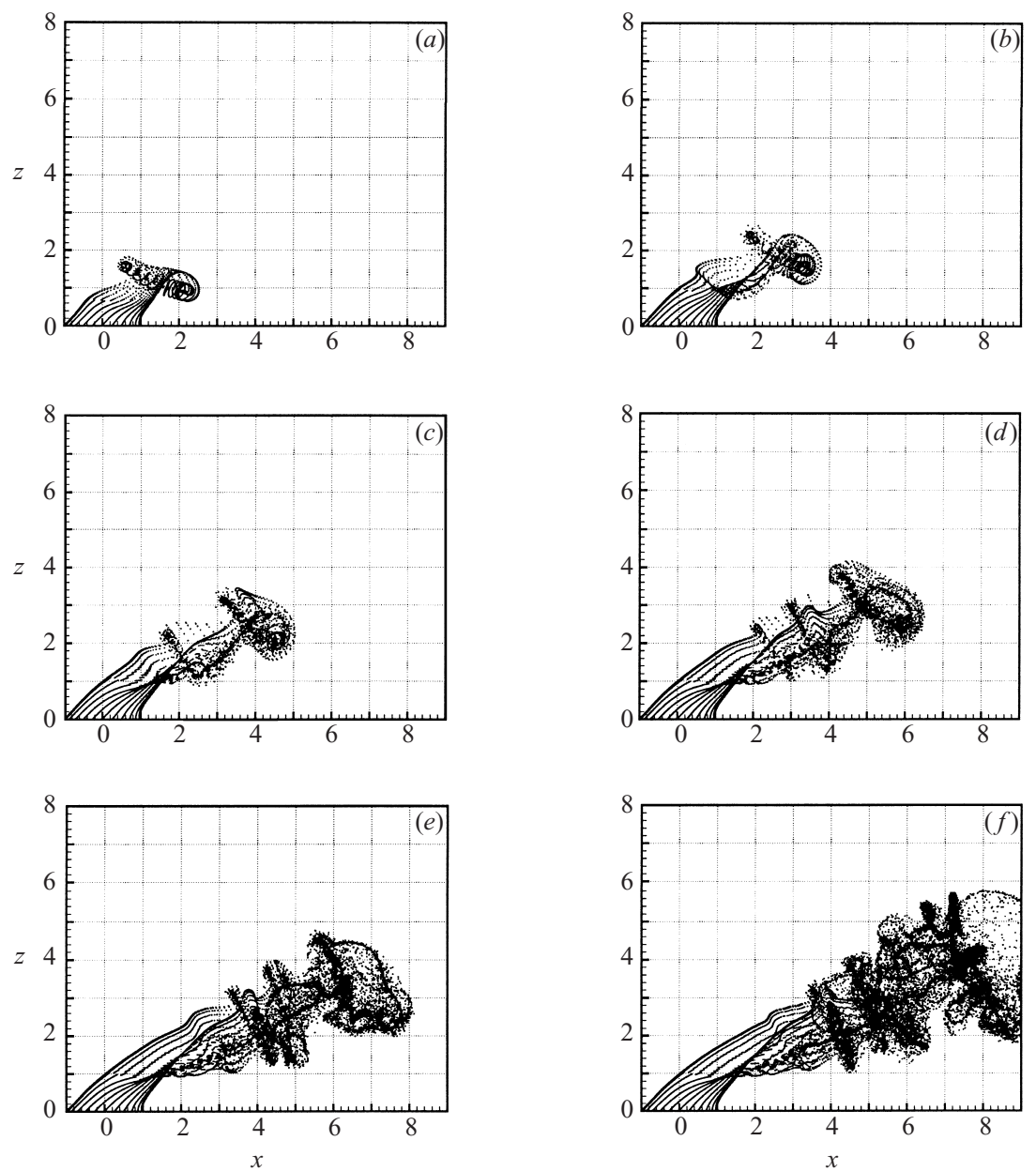

FIGURE 4. Side view of the evolution of the transverse jet: $U_{j e t} / U_{\infty}=2.5, \delta / D=0.5$, and $(a) t=3.0$, (b) 4.0, (c) 5.0, (d) 6.0, (e) 7.0, and $(f)$ 8.0. Vortex filaments with initially 24 nodes and core size $\sigma^{2}=0.1$ are introduced in the flow at each time step $\mathrm{d} t=0.02$.

on the temporal near-field evolution of the jet in crossflow. The effects of boundary layer thickness have not been explored experimentally, to the best of our knowledge, in part because it is difficult to modify an experimental apparatus in order to alter the effective boundary layer thickness upstream of the jet. Yet there have been experimental studies which have examined the effect of the location of transverse jet injection relative to a rearward-facing step placed upstream of the jet nozzle (e.g. Abbitt et al. 1993; Karagozian et al. 1996). The present results indicate that the boundary layer thickness, in many ways analogous to a rearward-facing step as used in high-speed engine configurations, can have a significant effect on jet evolution and penetration.

Figure 8 shows the side view of the evolution of a jet in a crossflow with successively increasing boundary layer thicknesses associated with the crossflow upstream of the jet. In these computations the non-dimensional jet velocity or mean velocity ratio $U_{\text {jet }} / U_{\infty}$ is maintained at 5.4. The evolution of the jet is represented by plotting the position of the nodes of the vortex filaments at a specific instant of time. 

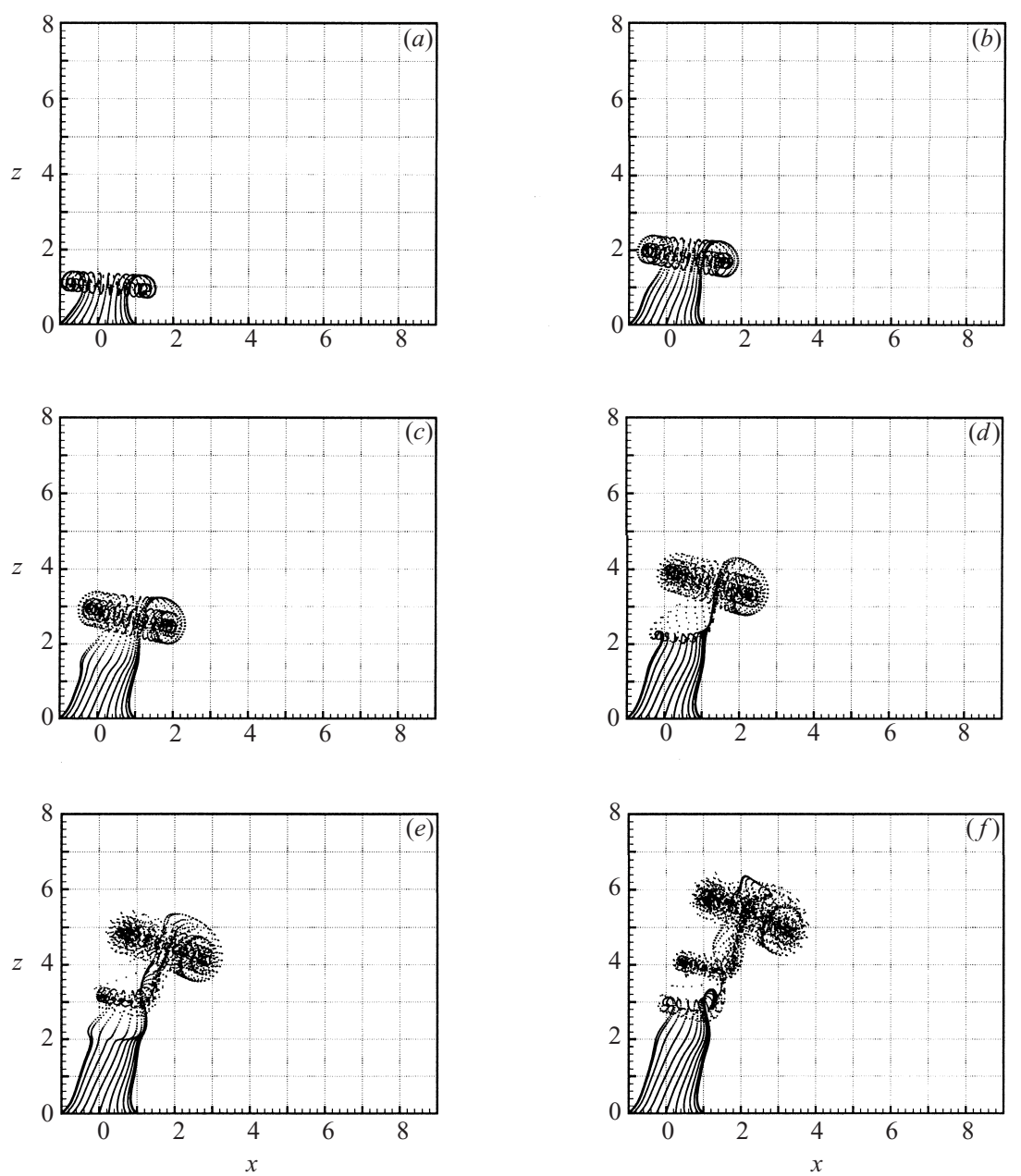

FIGURE 5. Side view of the evolution of the transverse jet: $U_{j e t} / U_{\infty}=10.8, \delta / D=0.5$, and (a) $t=0.75,(b) 1.00,(c) 1.25,(d) 1.50,(e) 1.75$, and $(f) 2.00$. Vortex filaments with initially 24 nodes and core size $\sigma^{2}=0.1$ are introduced in the flow at each time step $\mathrm{d} t=0.005$.

Figure $8(a-d)$ compares the structure of the jets evolving through boundary layers of doubling thicknesses at time $t=3.5$. The global trajectory of the jet is affected by the thickness of the boundary layer. The jet penetrates further (in the $z$-direction) into the crossflow with increasing boundary layer thickness, similar to the effects of increasing the height of an upstream rearward-facing step that are observed in experiments (Abbitt et al. 1993; Karagozian et al. 1996). Moreover, the tilt of the starting vortex ring with respect to the jet plane and the asymmetry (bending) during its rollup increases as the boundary layer thickness decreases. As noted above, the tilt angle and the asymmetry are responsible for the deformation of the cylindrical vortex sheet that connects the starting vortex ring to the jet exit. Consequently, the deformation of the cylindrical vortex sheet and subsequent formation of the secondary vortex ring are delayed in jets evolving through a thicker boundary layer.

Figure $8(a-d)$ also indicates that the frequency of formation of the secondary vortex rings can be influenced by the thickness of the boundary layer. The ring period of formation increases slightly with increasing boundary layer thickness, consistent with 


$\begin{array}{rll}U_{j e t} / U_{\infty} & \delta / D & T \\ 2.5 & 0.5 & 1.0 \\ 5.4 & 0.5 & 0.68 \\ 10.8 & 0.5 & 0.38 \\ 5.4 & 0.25 & 0.50 \\ 5.4 & 1.0 & 0.71 \\ 5.4 & 2.0 & 0.75\end{array}$

TABLE 1. Approximate values of the non-dimensional period of secondary vortex formation $T$ for different transverse jet conditions.

(a)

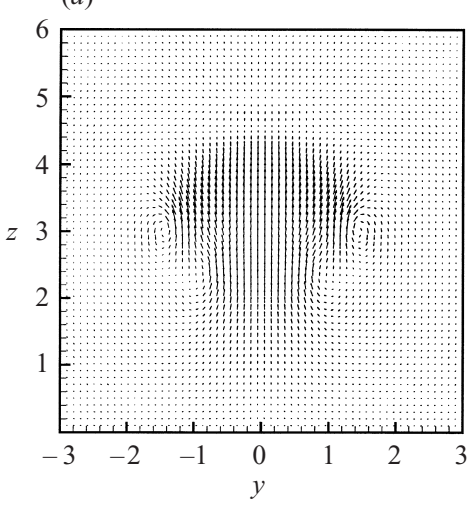

(c)

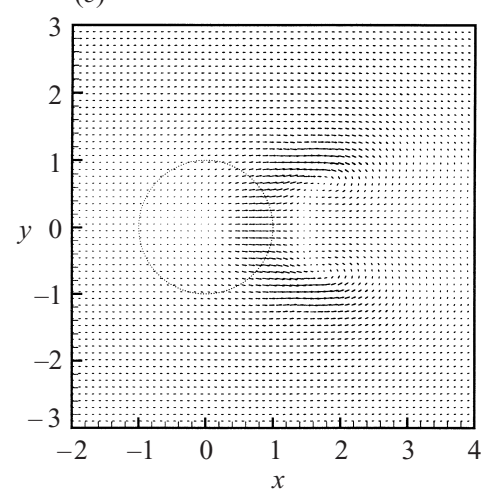

(b)

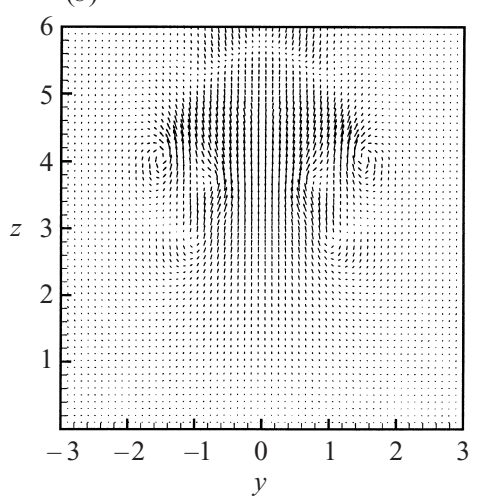

(d)

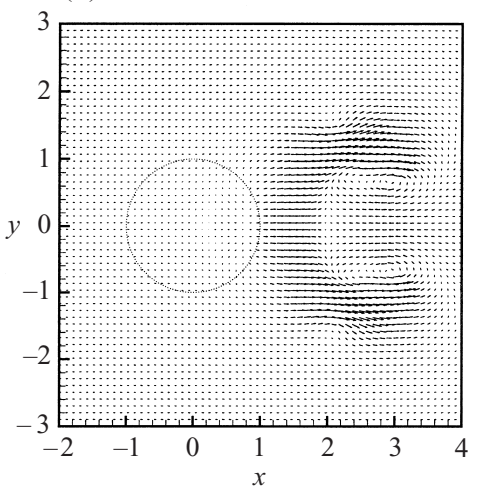

FIGURE 6. Velocity vector field at time $t=4.05$ in the $(y, z)$ plane, at $(a) x=2,(b) x=3$, and in the $(x, y)$-plane, at $(c) z=2,(d) z=3$, for the case $U_{j e t} / U_{\infty}=5.4, \delta / D=0.5$.

the delayed vortex ring folding and tilting. Table 1 compares the non-dimensional periods $T$ quantified for a few different cases, and also includes data from the other jet-to-crossflow velocity ratios described above. Not only does the characteristic period $T$ increase slightly with $\delta / D$ for a fixed $U_{\text {jet }} / U_{\infty}$, but it is observed that the period also remains more consistent over time for the larger boundary layer thicknesses than it does for the thinner boundary layers.

The above results suggest that jet penetration can be influenced by the jet-tocrossflow velocity ratio as well as by the upstream boundary layer thickness. Yet 
(a)

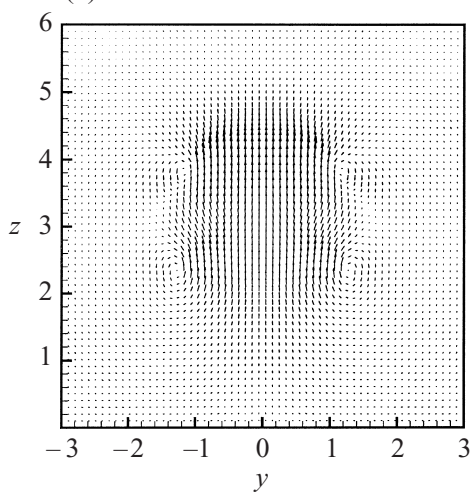

(c)

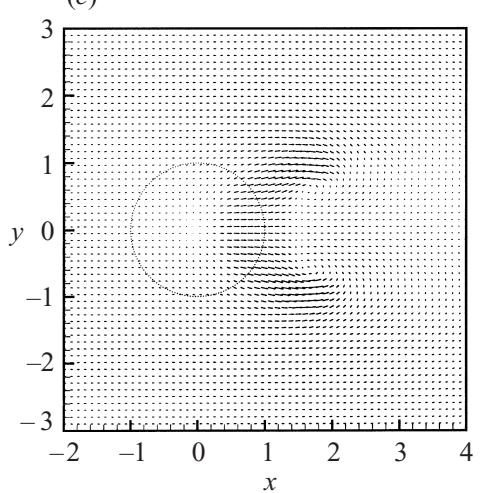

(b)

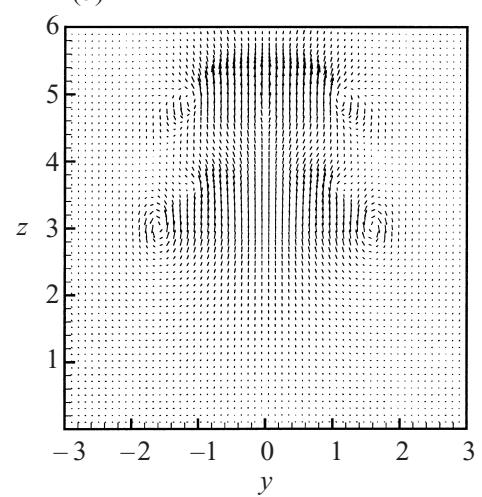

(d)

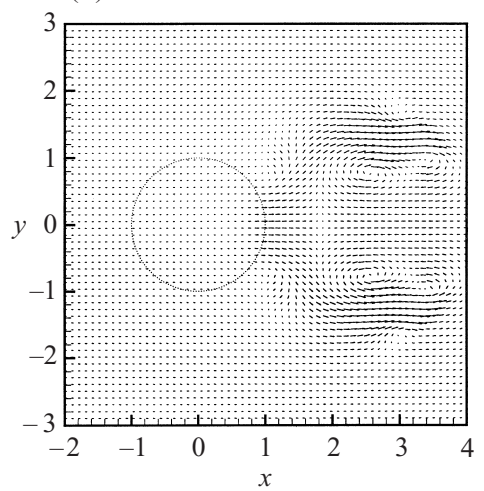

Figure 7. As figure 6 but at time $t=4.35$.

while penetration clearly increases with increasing boundary layer thickness and with $U_{j e t} / U_{\infty}$, the frequency of secondary vortex rollup (the inverse of $T$ ) does not appear to follow the same trend. The present simulations show that the deformation and interaction of successive vortex rings can be delayed (slightly increased $T$ ) with an increased boundary layer thickness, but that the period $T$ decreases with increasing $U_{j e t} / U_{\infty}$. The relevance of these phenomena to jet entrainment and mixing is less obvious, and a more detailed examination of entrainment, as elaborated in $\S 5$, is necessary.

But if the delay of vortex ring deformation and reduction in frequency of interaction is viewed as implying a reduction in the near-field jet-crossflow interaction and the rate of entrainment of crossflow into the jet, then the present results are generally consistent with limited experimental data. Systematic experiments involving planar laser-induced-fluorescence (PLIF) imaging of iodine-seeded transverse gas jets, injected behind a rearward-facing step (Karagozian et al. 1996), suggest that increasing the jet-to-crossflow velocity ratio, for a fixed step height and injection location, increases both jet penetration and mixing. If one considers the effect of the rearward-facing step upstream of the jet to be similar to that of a thick boundary layer, then the experimental findings are consistent with the present computational results. Moreover, in the experiments when the jet-to-crossflow velocity ratio is fixed and the jet injection location is moved closer to the upstream step, somewhat analo- 

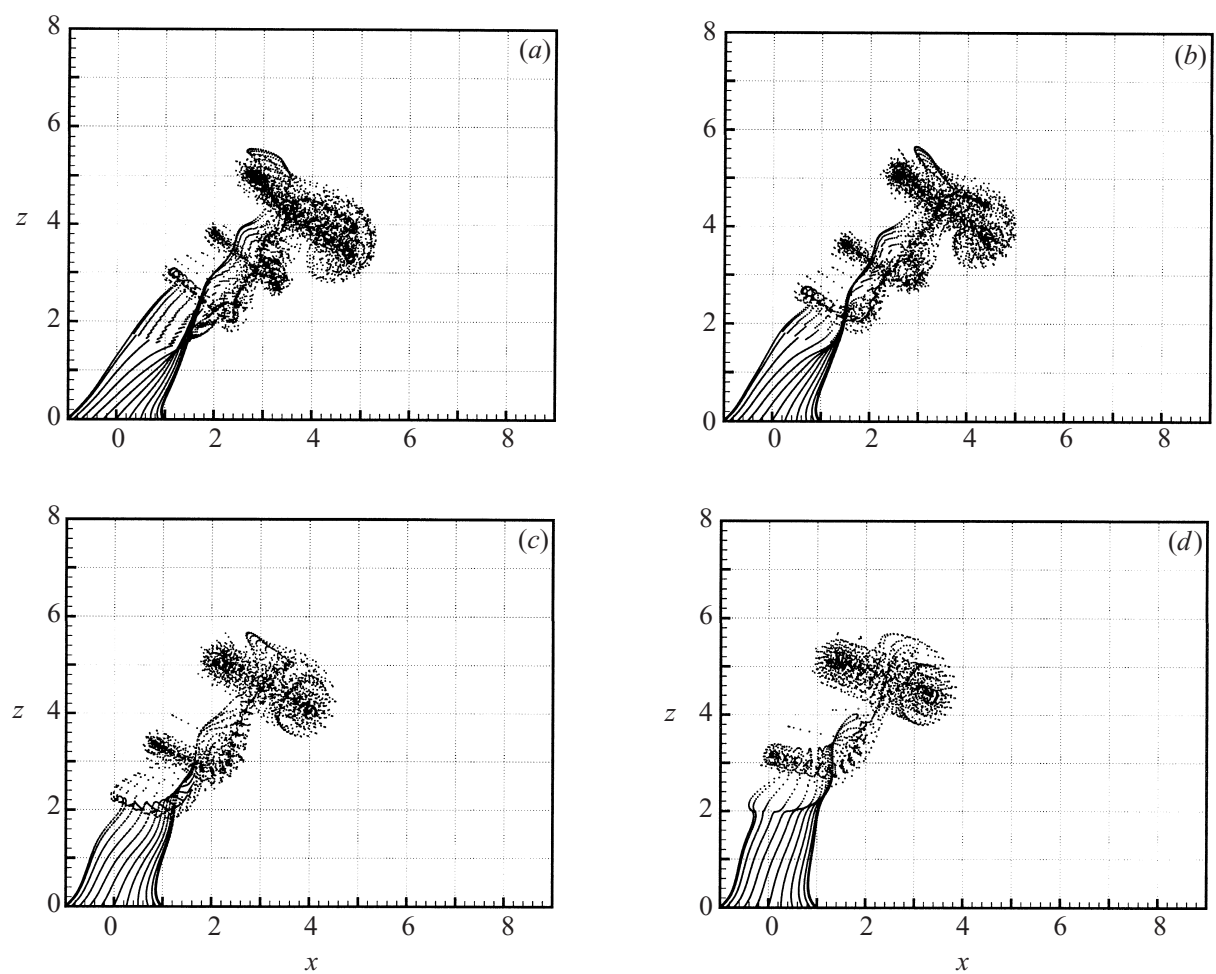

FIGURE 8. Effect of the boundary layer thickness on the evolution of the transverse jet (side view): $t=3.5, U_{j e t} / U_{\infty}=5.4$, with $(a) \delta / D=0.25,(b) 0.5,(c) 1.0$, and $(d) 2.0$.

gous to thickening the upstream boundary layer, the jet penetration increases but the degree of mixing between injectant and crossflow is actually diminished. Apparently when the degree of immediate interaction between the jet and crossflow is reduced or blocked, the jet has a chance to penetrate further in the near field, but because of this reduced interaction with crossflow, the actual near-field entrainment and mixing may be reduced.

A word should also be said about the quantification of the non-dimensional characteristic period $T$ associated with vortex (or shear layer) rollup for the nonforced jet in crossflow. Characteristic periods have not been extensively measured in experimental studies of the transverse jet. Kelso et al. (1996) suggest from gasphase experiments that the unforced shear layer on the upstream side of the jet, within three diameters of the jet exit, rolls up with a characteristic frequency of $7.1 \mathrm{~Hz}$. This corresponds to a Strouhal frequency based on crossflow velocity of $S t_{\infty} \equiv f D / U_{\infty}=0.65$. Kelso et al. (1996) also performed experiments in which acoustic excitation of the jet was applied at this 'characteristic' frequency $7.1 \mathrm{~Hz}$, and extracted phase-locked vorticity contours in the flow field. Other experimental investigations of the pulsed jet in crossflow, either carried out in liquid (Eroglu \& Breidenthal 1991; Hermanson, Wahba \& Johari 1998; Johari, Pacheco-Tougas \& Hermanson 1999) or in gas (Schuller et al. 1999) used frequencies of excitation that were much lower than that by Kelso et al. (1996), when quantified in terms of Strouhal numbers based on either jet or crossflow velocity. In fact, Schuller et al. (1999) found that external excitation of the jet at frequencies well below that of the 'characteristic' 
rollup frequency causes the jet penetration and spread to increase substantially above that for the unforced jet.

Yet for the jet in crossflow problem, a Strouhal frequency based only on diameter and either jet or crossflow velocity is likely not to be an appropriate 'non-dimensional' characterization of the rollup process, since the dynamics of the rollup of the shear layer are dependent on both crossflow and jet velocities, as well as the injection wall boundary layer thickness, jet diameter, and jet nozzle characteristics. At the very least, a Strouhal frequency which takes into account both characteristic velocities needs to be defined. This analysis is beyond the scope of the present study, although one possible estimate may simply be a Strouhal frequency based on an average of the mean jet and crossflow velocities: $S t_{\text {mean }} \equiv 2 f D /\left(U_{\text {jet }}+U_{\infty}\right)$. On this basis, the value of $S t_{\text {mean }}$ extracted from vortex rollup in the present computations, say for the baseline case of $U_{\text {jet }} / U_{\infty}=5.4$ and $\delta / D=0.5$, is 0.92 . For $U_{\text {jet }} / U_{\infty}=2.5$ and 10.8 , the values of $S t_{\text {mean }}$ are 1.14 and 0.89 , respectively. Comparable experimental values of the characteristic $S t_{\text {mean }}$ for unforced jets in the studies of Schuller et al. (1999) and Kelso et al. (1996) are 0.77 and 0.40, respectively. While this 'simple' determination may yield a rough estimate of a scaled frequency, it does not account for variation in the upstream boundary layer thickness, which also affects rollup. Again, because the vortex rollup process is so intimately related to a variety of parameters which characterize the transverse jet, a more appropriate parameter needs to be defined for adequate comparisons to be made and appropriate trends (as in table 1) to be more accurately delineated.

\section{Formation of the counter-rotating vortex pair}

To reconcile the temporal evolution of the vorticity field with experimental observations of the CVP, we attempt to simulate the averaging process commonly used in experiments. We compute the three-dimensional velocity field at different times during a given cycle associated with the formation of a secondary vortex ring. Subsequently, we compute the ensemble average of the velocity fields over this cycle and, from the averaged velocity field, we compute the associated averaged vorticity field. The ensemble over which we perform the average, 13 measurements, is small with respect to the ensembles usually used in experiments, which may involve hundreds of measurements, yet due to the present use of three-dimensional Lagrangian vortex dynamics, the current averaging process is in fact able to reconstruct an accurate time-averaged structure of the jet. When the number of realizations within each ensemble average is either doubled or halved, there is no apparent difference in results.

Figure 9 shows the ensemble averaging of the velocity vector field for the jet in crossflow where $U_{j e t} / U_{\infty}=5.4$. The time (ensemble) averaging of the velocity vector field is associated with 13 instantaneous realizations of the jet in crossflow at nondimensional times ranging from $t=4.0$ to 4.60 , inclusive. Figures $9(a)$ and $9(b)$ show the averaged velocity vector field in the $(y, z)$-cross-plane at the downstream locations $x=2$ and 3, while figures $9(c)$ and $9(d)$ present the averaged velocity vector field looking down on the jet, in the $(x, y)$-plane parallel to the jet-exit plane at the locations $z=2$ and 3 . The dotted line in figure $9(c, d)$ shows the location of the jet exit at $z=0$. Both views of the average jet velocity field elucidate the initiation of a CVP structure, and in fact produce similar velocity field representations to those extracted via laser-Doppler velocimetry measurements by Kamotani \& Greber (1972). The fact that vortex cores are visualized in both planes is an indication of the threedimensional character of the actual CVP. The CVP structure is much more clearly 

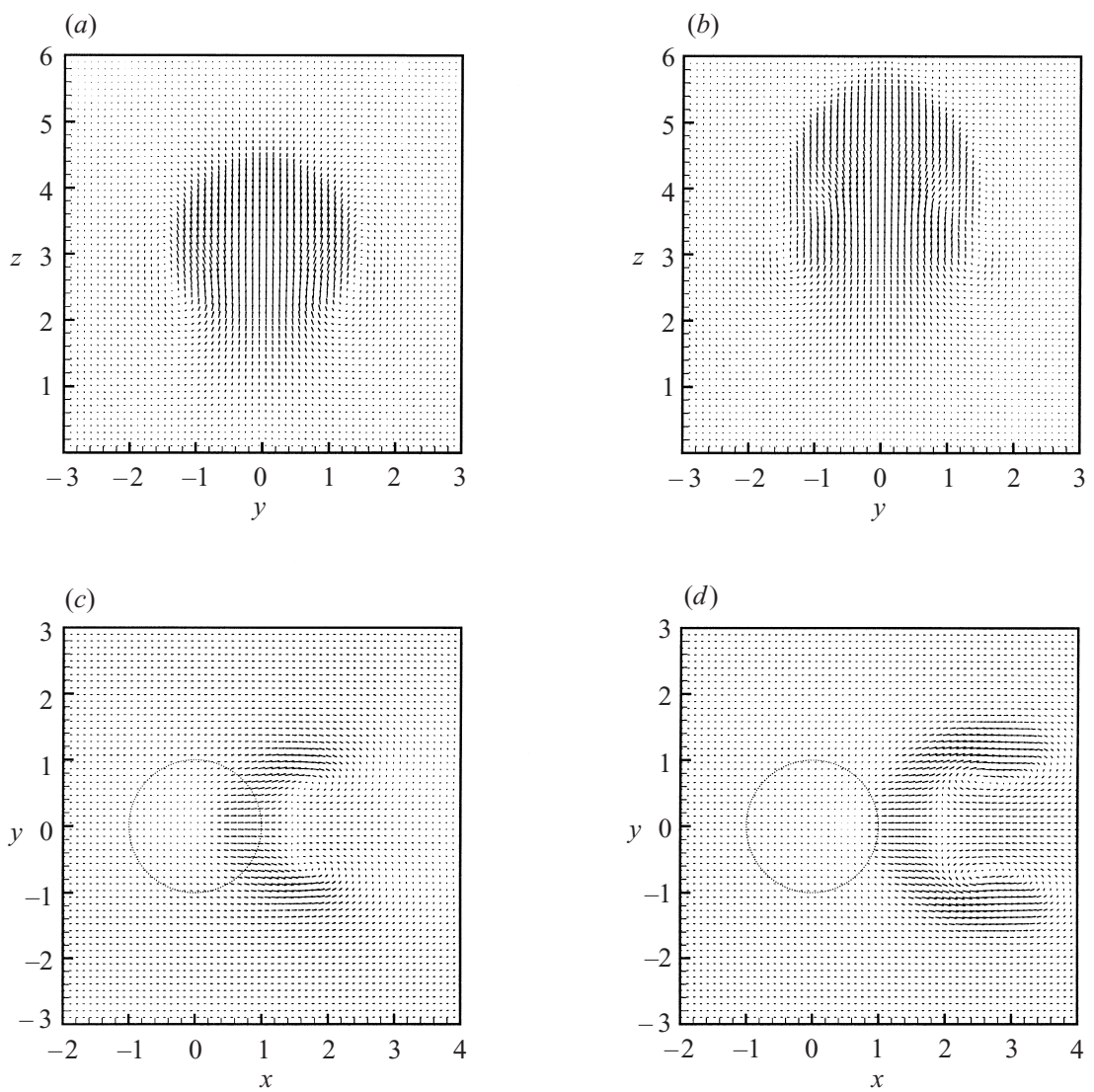

FIGURE 9. Ensemble-averaged velocity vector field in the $(y, z)$-plane, at $(a) x=2,(b) x=3$, and in the $(x, y)$-plane, at $(c) z=2,(d) z=3$, for the case $U_{j e t} / U_{\infty}=5.4, \delta / D=0.5$.

defined in these averaged velocity vector images than in the instantaneous images (figures 6 and 7).

To visualize the three-dimensional nature of the initiation of the CVP, we compute the averaged vorticity from the time (ensemble) averaging of the velocity vector field, presented in figure 9. Figure 10 shows a three-dimensional view of the magnitude of the averaged vorticity field for the jet in crossflow where $U_{\text {jet }} / U_{\infty}=5.4$. The light grey surface corresponds to the isosurface of the magnitude of the averaged (nondimensional) vorticity field, $\bar{\omega} \equiv \sqrt{\omega_{x}^{2}+\omega_{y}^{2}+\omega_{z}^{2}}$ equal to 4.0. This isosurface may be designated as that which separates regions of 'high' and 'low' vorticity for these specific operating conditions. This light grey isosurface is in fact nearly identical to the isosurfaces where the RMS value of the cross-product of velocity and vorticity vectors is minimized. Hence the $\bar{\omega}=4.0$ isosurface is likely to be a reasonable representation of the 'jet' region, although at $z=0$ this isosurface lies somewhat external to the actual jet orifice due to the boundary conditions employed. The regions in which the magnitude of the vorticity vector is greater than 30 (shown in darker grey in figure 10) have a strong similarity to the jet structure observed experimentally when jet fluid is seeded with dye (Kelso et al. 1996). High-magnitude vorticity is clearly contained within two slim columns which resemble the CVP cores. Figure 11(a-d) shows 'exploded' views of some of the separate vorticity isosurfaces seen in darker 


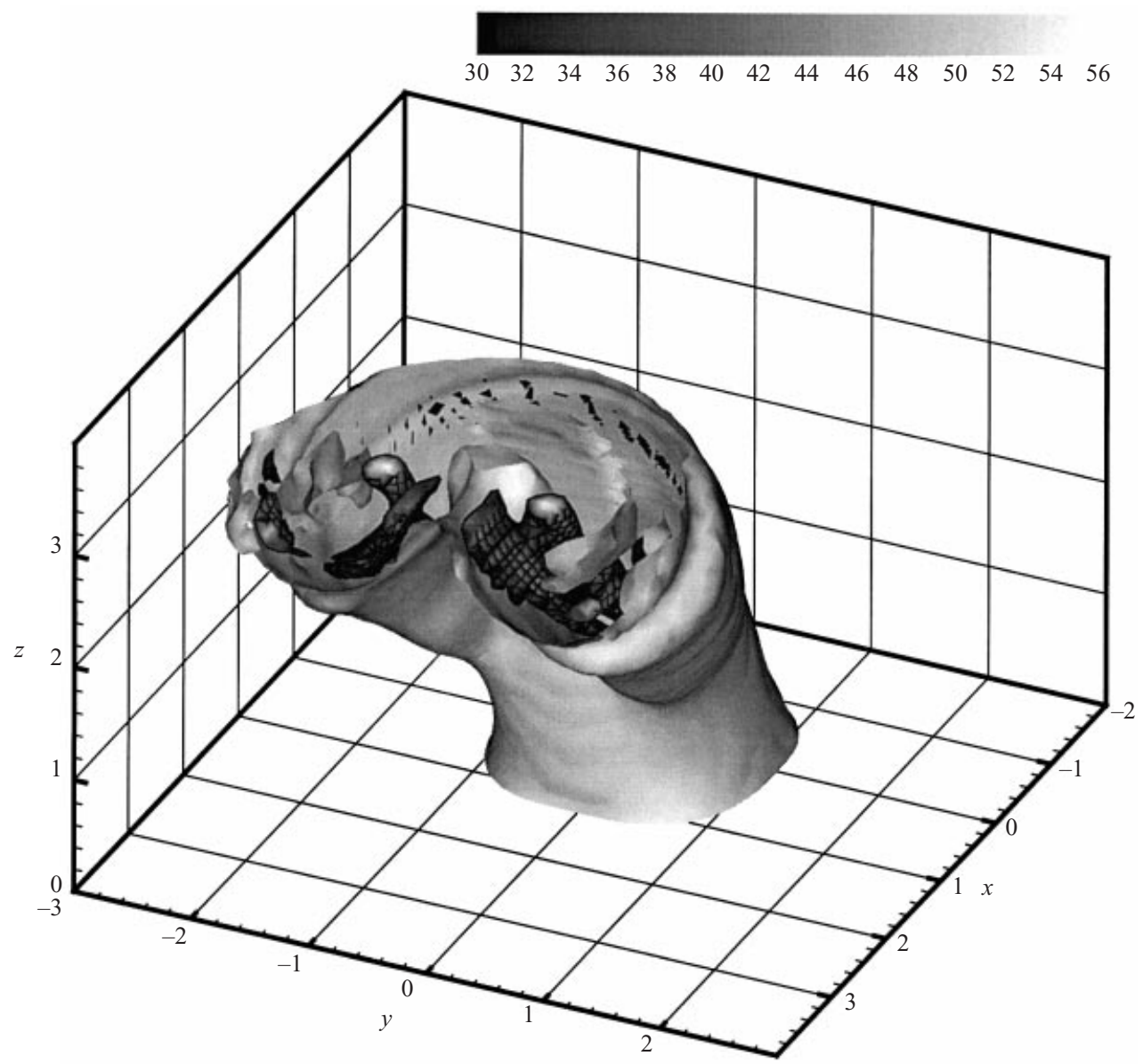

FIGURE 10. Ensemble-averaged magnitude of the vorticity field for the case $U_{\text {jet }} / U_{\infty}=5.4, \delta / D=0.5$. The light grey outer isosurface corresponds to average vorticity magnitude $\bar{\omega}=4.0$, while darker isosurfaces correspond to values of $\bar{\omega}$ of 30 and above, as delineated in the grey scale map shown. The cross-hatching at the lower vorticity values $(30-38)$ is an artifact of the plotting routine used here.

grey in figure 10 , for the values $\bar{\omega}=25,27,30$ and 32 , respectively. The origins of the initiation of the CVP are evident as rollup and bending of the vortex sheet occurs (figure 11a), leading to tight columnar vorticity isosurfaces (figure $11 b$ ) which originate even within one radius of the jet orifice. Strengthening of this rollup is evident as one moves further away from the injection plane (figure $11 c, d$ ). These robust vortical structures, which form independently of the alternative computational boundary conditions explored, appear to be the initiation of the fully developed CVP structure seen experimentally in the far field. This near-field initiation of the CVP is suggested by a number of recent experiments (Kelso et al. 1996; Smith \& Mungal 1998).

The ensemble averaging here also enables validation of the ideas of Kelso et al. (1996) with respect to initiation of the CVP via folding and tilting of the secondary vortex ring which enters the flow field. Figure $12(a, b)$ shows three-dimensional and side views, respectively, of the instantaneous positions of vortex filaments for the $U_{\text {jet }} / U_{\infty}=5.4$ case, as done in figure $3(f)$ at time $t=4.0$, on top of which are 

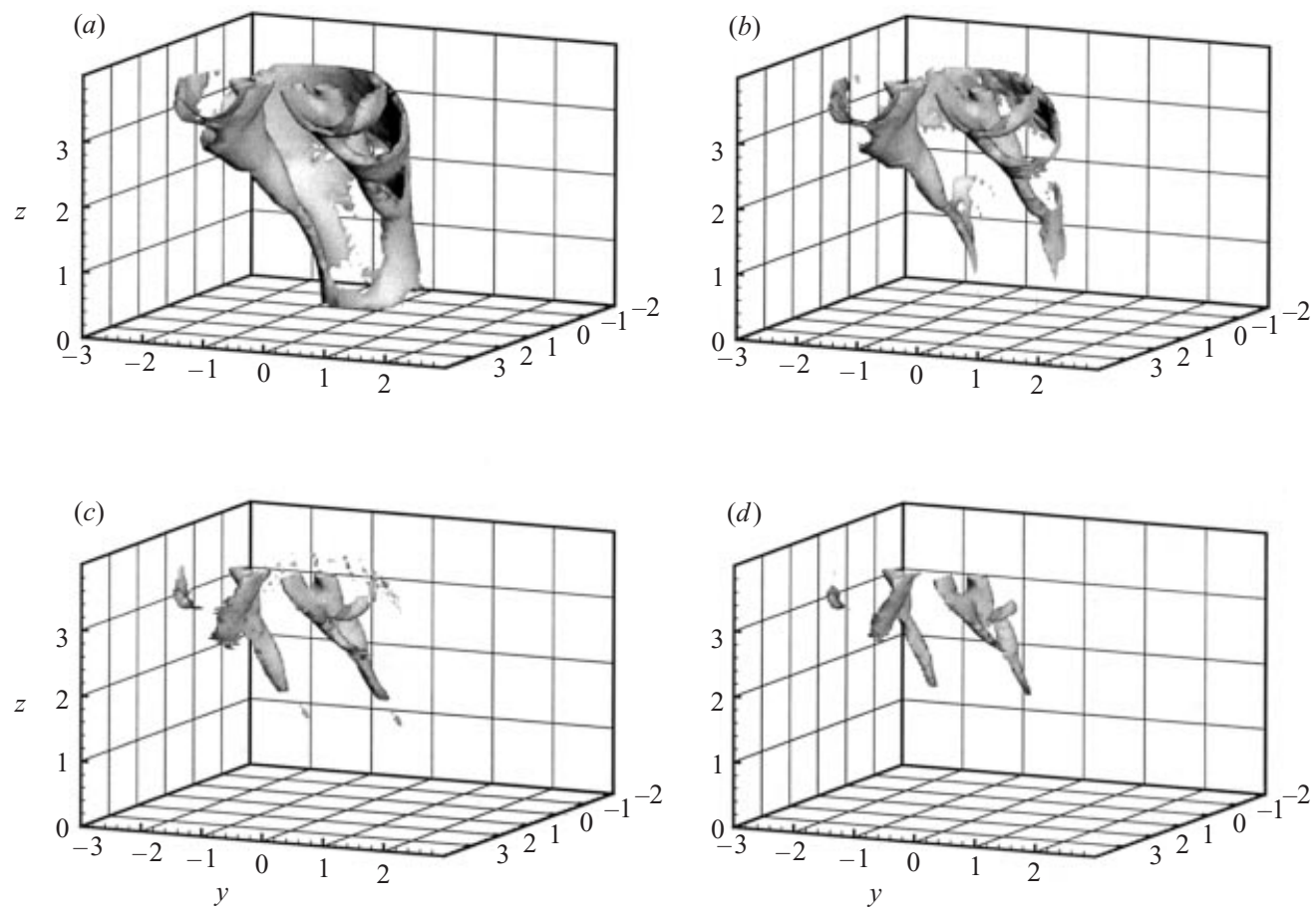

FIGURE 11. 'Exploded view' of the ensemble-averaged magnitude of the vorticity field for the case $U_{\text {jet }} / U_{\infty}=5.4, \delta / D=0.5$. The average vorticity isosurfaces correspond to $(a) \bar{\omega}=25,(b) \bar{\omega}=27$, (c) $\bar{\omega}=30$, and $(d) \bar{\omega}=32$.

superposed the grey, ensemble-averaged three-dimensional vorticity isosurfaces $\bar{\omega}=$ 30. As noted in figures 10 and 11 , the $\bar{\omega}=30$ contour appears to enclose the cores of the evolving three-dimensional CVP; in figure $12(a, b)$ this contour is clearly coincident with the 'downstream part' of the tilted secondary vortex ring, similar to concentrated dye evolution visualized in the experiments of Kelso et al. (1996) and more recently in Lim, Kelso \& Perry (1998). Thus there is strong evidence that, as proposed by Kelso et al. (1996), the initial bending of the vortex sheet is the first sign of the initiation of the CVP structure.

\section{Crossflow entrainment by the transverse jet}

Among the benefits of using the jet in crossflow in a gas turbine engine environment is the enhancement of the entrainment of crossflow into the vicinity of the jet and the subsequent enhancement of the molecular mixing of the two fluids that occurs. Entrainment by a transverse jet or flame is typically seen to be much greater (see Broadwell \& Breidenthal 1984; Brzustowski 1977; Karagozian 1986b; Smith \& Mungal 1998) than that by a free jet (Hottel \& Luce 1953) or a buoyant turbulent jet (Steward 1970), for example. Smith \& Mungal (1998) suggest that, in the light of the evolving development of the CVP in the near field of the jet, the enhanced mixing in the jet's near field corresponds to and likely results from the structural formation of the CVP rather than its fully developed existence. This near-field enhancement of entrainment and the potential for enhancement of molecular mixing may be explored in the present vortex element simulations. 
(a)

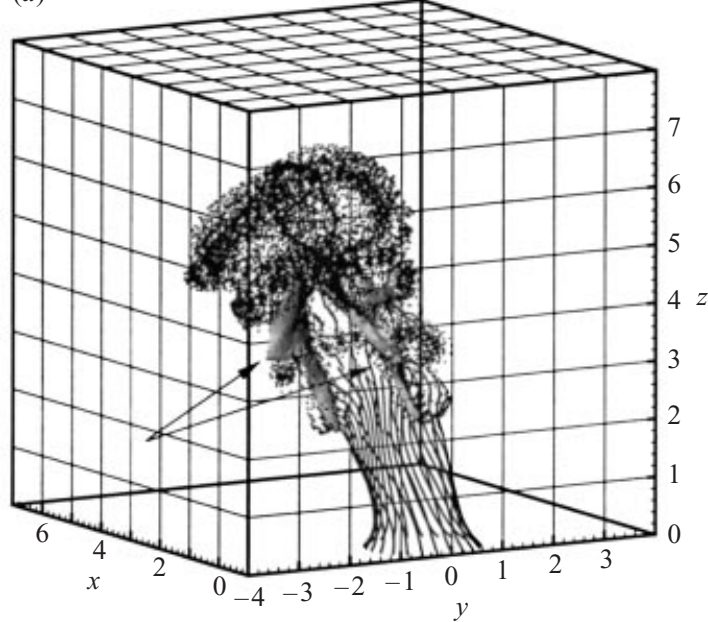

(b)

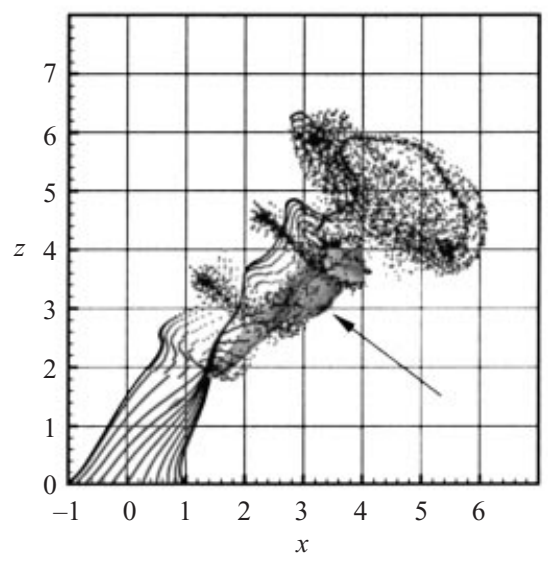

FIGURE 12. Instantaneous positions of the transverse jet: $U_{\text {jet }} / U_{\infty}=5.4, \delta / D=0.5$, at time $t=4.0$, with $(a)$ three-dimensional view and $(b)$ side view, as in figure 3. Superposed on these plots is the ensemble-averaged three-dimensional vorticity isosurface $\bar{\omega}=30$, shown in grey and indicated by the arrows.

The present simulations allow a means for detailed examination of near-field entrainment because the ensemble averaging of the velocity vector field is by definition time-independent. Consequently, the entrainment of the crossflow can be characterized using stream-tracers imposed in the ensemble-averaged flow field here, since streamlines and streaklines coincide. Figure 13 shows the light grey vorticity isosurface $\bar{\omega}=4.0$ for the jet in crossflow with velocity ratio $U_{j e t} / U_{\infty}=5.4$, as done in figure 10 , on which there is superposed the evolution of a densely packed array of smoke tracers initially placed two radii upstream of the jet centreline. The yellow streaklines enclosed within the upstream portion of the vorticity isosurface are associated with fluid originating within the jet nozzle, which was initially distributed uniformly within the circular jet orifice at $z=0$. The other coloured streaklines represent crossflow fluid originating upstream of the jet, initially situated within one radius (purple), two radii (pink), three radii (orange), and four radii (green) of the injection wall. These streakline patterns indicate that the crossflow is deflected about the jet, and then can be strongly entrained into the jet on its lee side, with streaklines winding into the vortical structures which form in the ensemble-averaged flow field. The concentration of the yellow jet fluid within the upstream portion of the $\bar{\omega}=4.0$ vorticity isosurface, above the bulk CVP structure, is consistent with experimental findings dating from the work of Kamotani \& Greber (1972). These and subsequent experimental studies suggest that the local centre of the CVP structure spatially 'lags' or lies below the local maximum in axial jet velocity. Most of the crossflow streaklines shown in figure 13, even those originating close to the injection wall (purple), are lifted up on the lee side of the jet into the centreline region of the evolving vortex pair structure, later winding into the vortices themselves.

This latter observation is also consistent with those of Fric \& Roshko (1994) as well as Smith \& Mungal (1998), which suggest that the upright wake vortices forming downstream of the transverse jet originate in the wall boundary layer. As the purple streaklines originating in the wall boundary layer are deflected about the jet, they fan outward and upward into the evolving CVP associated with the jet fluid. 


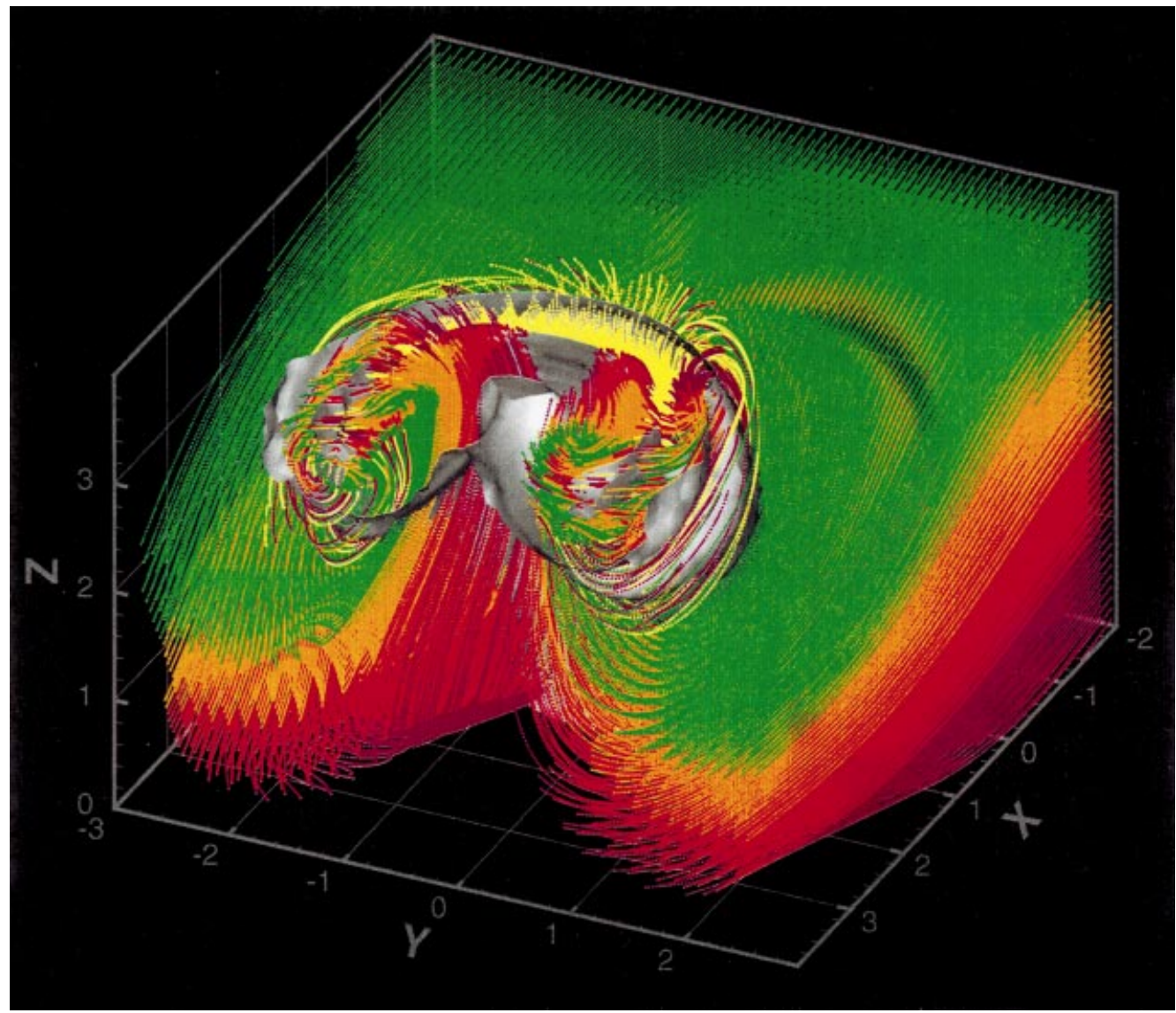

FiguRE 13. Averaged magnitude of the vorticity field $\bar{\omega}=4.0$ for the case $U_{\text {jet }} / U_{\infty}=5.4, \delta / D=0.5$, on which is superposed the evolution of horizontal arrays of 'smoke' tracers. The tracer streaklines are computed from the ensemble-averaged velocity field. The yellow streaklines are associated with fluid originating within the jet nozzle, while the other coloured streaklines represent crossflow fluid originating upstream of the jet, at $x=-2$, within one radius (purple), two radii (pink), three radii (orange), and four radii (green) of the injection wall.

This phenomenon is also similar to the lifting up of boundary layer dye into the jet observed in the experiments of Kelso et al. (1996), which led these researchers to postulate the existence of a saddle-node point on the lee side of the jet exit. This three-dimensional critical point is a saddle in the jet plane and a node in the symmetry plane. As the streamlines approach the critical point, they stretch in the jet plane due to the saddle point and fan out in the symmetry plane due to the node creating a stream-surface. Since the region of our computed saddle point is far enough downstream of jet injection, we believe that the present simulations provide an accurate rendering of this phenomenon. A more comprehensive topological map of the flow near the jet exit may be found in the discussion by Kelso et al. (1996).

Figure 13 also suggests that, far enough away from the jet near-field structure (in the $y$-direction), smoke tracers can completely bypass the jet, apparently avoiding any substantial degree of entrainment into the jet. This observation is further demonstrated in figure 14, which shows smoke tracers initially situated four radii downstream of 


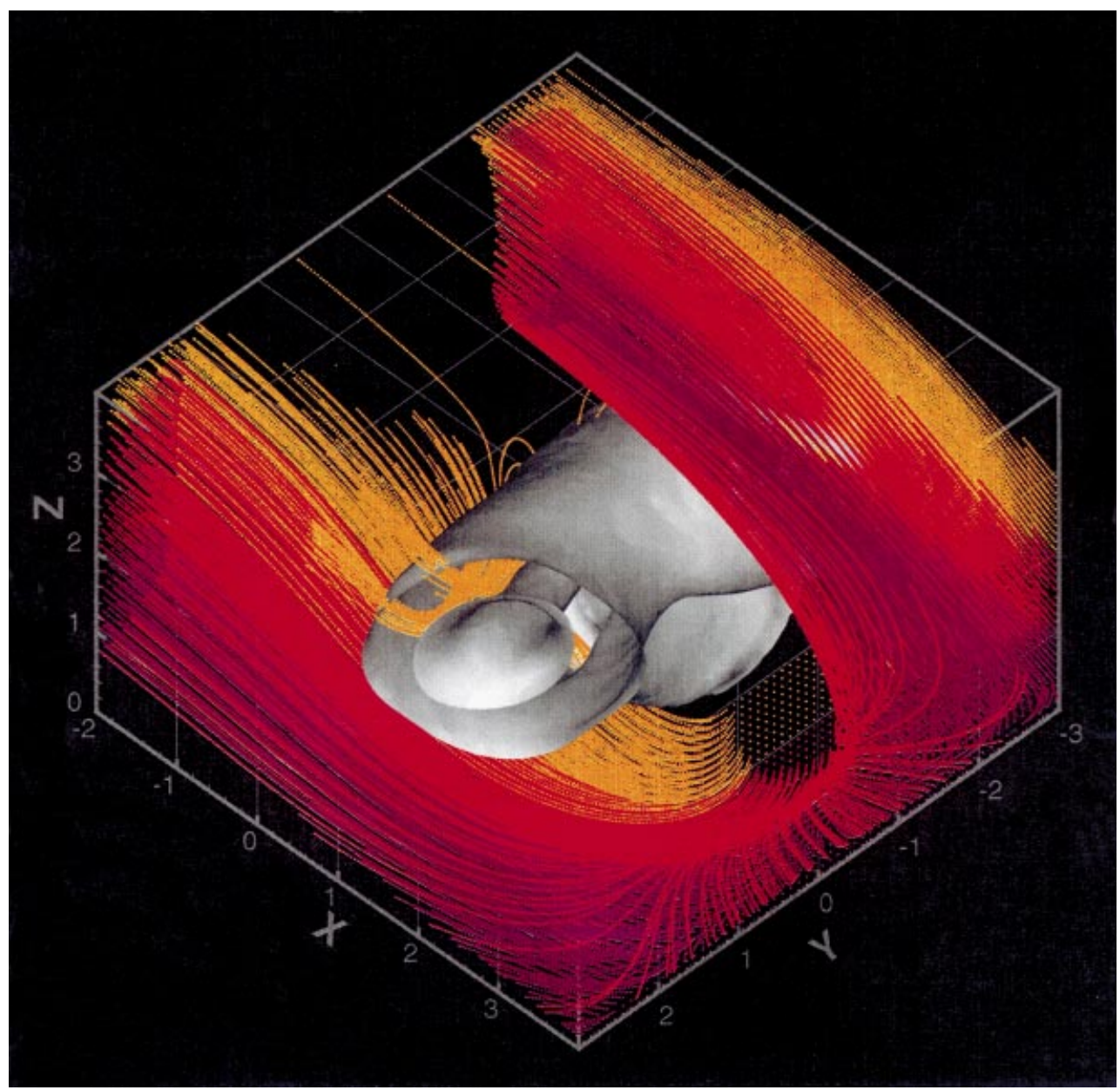

FIGURE 14. Averaged magnitude of the vorticity field for the case $U_{j e t} / U_{\infty}=5.4, \delta / D=0.5$, on which is superposed the backward evolution of arrays of 'smoke' tracers originating outside of the jet at $x=4$. The tracer streaklines shown are those, computed from the averaged velocity field, which have bypassed the jet structure and are not entrained into the CVP.

and external to the jet, at $x=4$, with the same colour scheme as in figure 13 in the region $0 \leqslant z \leqslant 3$. A 'backward' tracing of the origination of the smoke is made, for this ensemble-averaged flow field, to the location $x=-2$. Virtually all of these 'external' streamtracers correspond to streaklines which originated in the crossflow, indicating that there has been a complete bypassing of the jet. Such streaklines show evidence of a bathtub-shaped stream-surface surrounding the jet near field which contains crossflow fluid that is entrained into the jet. All the crossflow fluid outside of this bathtub-shaped stream-surface completely bypasses the jet.

The evolution of streamtracers within the ensemble-averaged transverse jet flow field also provides data from which entrainment of crossflow by the jet may be visualized. Figure $15(a-c)$ shows a series of slices of the averaged jet flow field in the $(x, y)$-plane, at different $z$-locations, which measure non-dimensional distance from the injection wall ( $z=1,2$, and 3 , respectively). These slices, of thickness $\Delta z=0.04$, reveal the $\bar{\omega}=4.0$ vorticity isosurface, which at the injection wall lies somewhat external to 

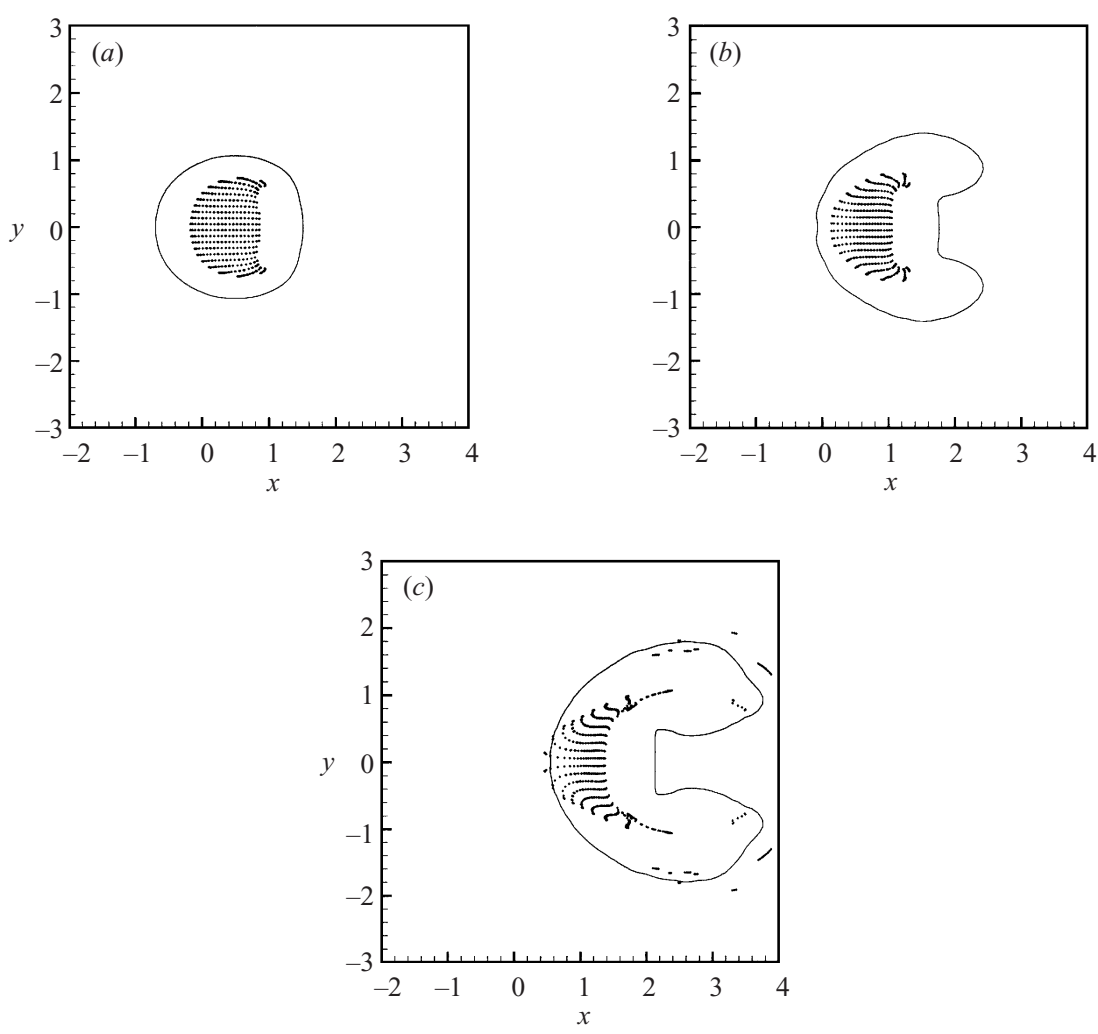

FIGURE 15. Slices in the $(x, y)$-plane of the transverse jet evolution: $U_{j e t} / U_{\infty}=5.4, \delta / D=0.5$, at $z$-locations $(a) 1.0,(b) 2.0$, and $(c) 3.0$. A slice of the average vorticity isosurface $\bar{\omega}=4.0$ is shown. Also shown, in black, are the positions of streamtracers which originated within the jet, corresponding to tracer positions shown in yellow in figure 13 .

the jet orifice. Also shown are the positions of streamtracers which originated within the jet (shown in black; these were yellow in figure 13). The evolution of the flow field in the $z$-direction provides further visual evidence for the entrainment of crossflow as the transverse jet and associated CVP develop. As $z$ increases, the region containing black (jet) streamtracer particles proportionately decreases within the area enclosed by the vorticity isosurface.

Consistent with the visualization in figure 13 , as the crossflow is entrained into the jet in figure 15, principally on the lee side, jet fluid tends to become concentrated within the upstream portion of the deformed 'jet slice'. Figure 15(c) further suggests that a small amount of jet fluid can be ejected out of the vorticity isosurface downstream of injection, as more and more of the jet region is composed of crossflow fluid. At the location $z=3$, the jet fluid itself comprises less than one third of the fluid contained within this slice of the vorticity isosurface. In fact, the jet fluid's near-field deformation in the $(x, y)$-plane, evolving from a circle to the cusped structures seen by the tracers in figure $15(a-c)$, is remarkably similar to that shown by Kelso et al. (1996) as an interpretation of the reorientation processes for the jet shear layer (see figure $2 b$ ). The association of vortex tilting and folding with entrainment into the jet is thus further visualized in figure $15(a-c)$.

As noted previously, jet penetration increases with increasing jet velocity and with boundary layer thickness, but the characteristic period of vortex rollup does 

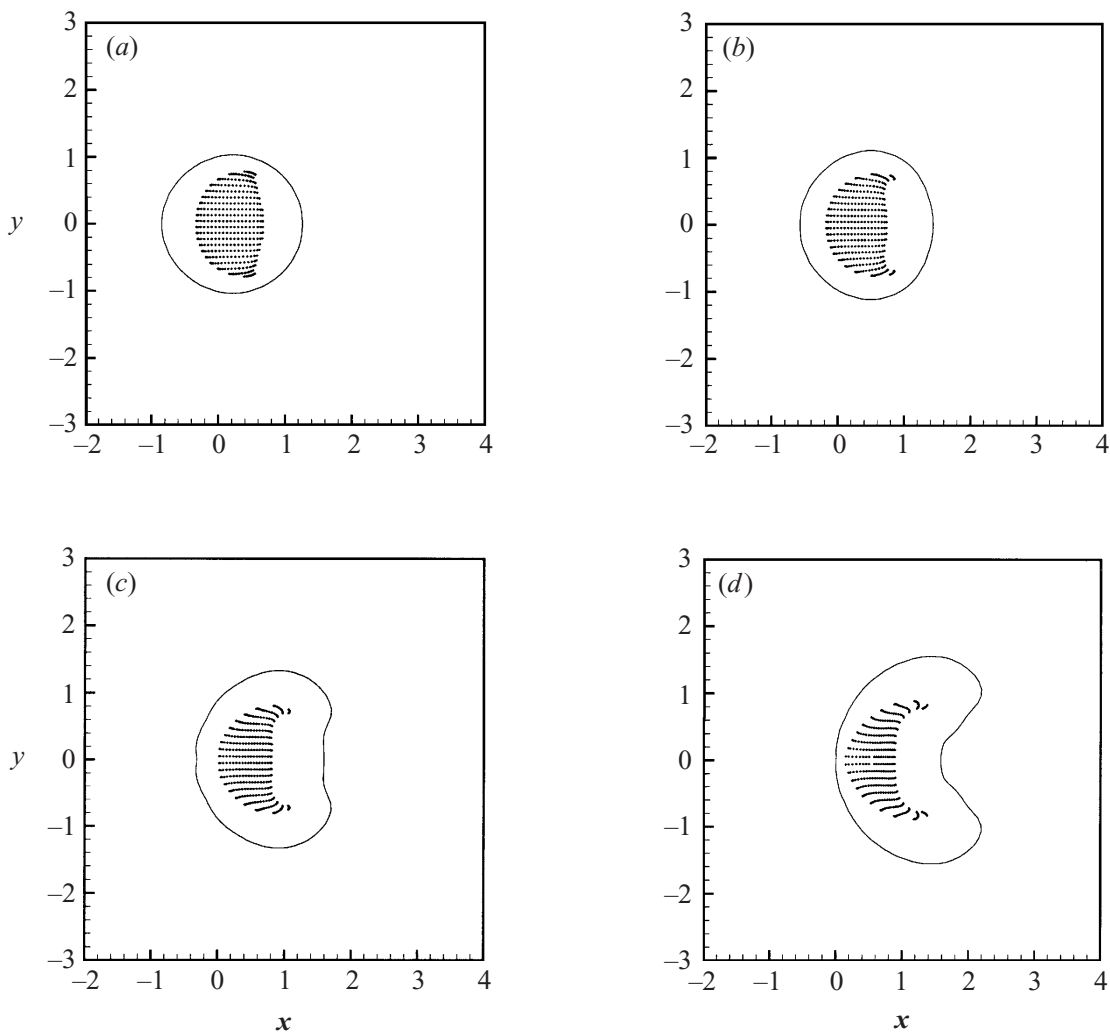

FiguRE 16. Slices in the $(x, y)$-plane of the transverse jet evolution: $U_{j e t} / U_{\infty}=10.8, \delta / D=0.5$, at $z$-locations $(a)$ 1.0, (b) 2.0, (c) 3.0, and (d) 4.0. A slice of the average vorticity isosurface $\bar{\omega}=10$ is shown. Also shown, in black, are the positions of streamtracers which originated within the jet.

not correlate directly with jet penetration. To see the implications of penetration and rollup frequency on the visualization of crossflow entrainment, we also explore the evolution of jet slices for other operating conditions. Figure $16(a-d)$ shows the evolution of jet slices and the positions of jet streamtracer particles, as done in figure 15 , but for the jet-to-crossflow velocity ratio 10.8 , keeping $\delta / D=0.5$. Similarly, figure $17(a-d)$ shows jet slices for the jet-to-crossflow velocity ratio 5.4 , but with the upstream boundary layer thickness increased to $\delta / D=2.0$. The slices of the vorticity isosurfaces shown correspond to $\bar{\omega}$ values which, as before, minimize the cross-product of the local velocity and the vorticity vectors. That $\bar{\omega}$ is higher in figure 16 and lower in figure 17 indicates the stronger and weaker CVPs, respectively, initiated for these different flow conditions. Interestingly, the ensemble-averaged jet slices for $z=1,2$ and 3 in figures $16(a-c)$ and $17(a-c)$ are remarkably similar. The effects of increasing jet momentum and increasing boundary layer thickness appear to have similar effects on the very near-field entrainment of crossflow into the vorticity isosurface, which appears to proceed more slowly than for the 'baseline' flow conditions in figure $15(a-c)$. The significant deformation of the isosurface slice seen in figure $15(c)$ at $z=3$ is not realized in the thicker boundary layer case until $z=4$ (figure $17 d$ ) and even later for the higher jet momentum case (figure 16). These results suggest that the increased jet penetration seen for higher jet-to-crossflow velocity ratios (figures 5 and 16) and for greater boundary layer thicknesses (figures $8(d)$ and 

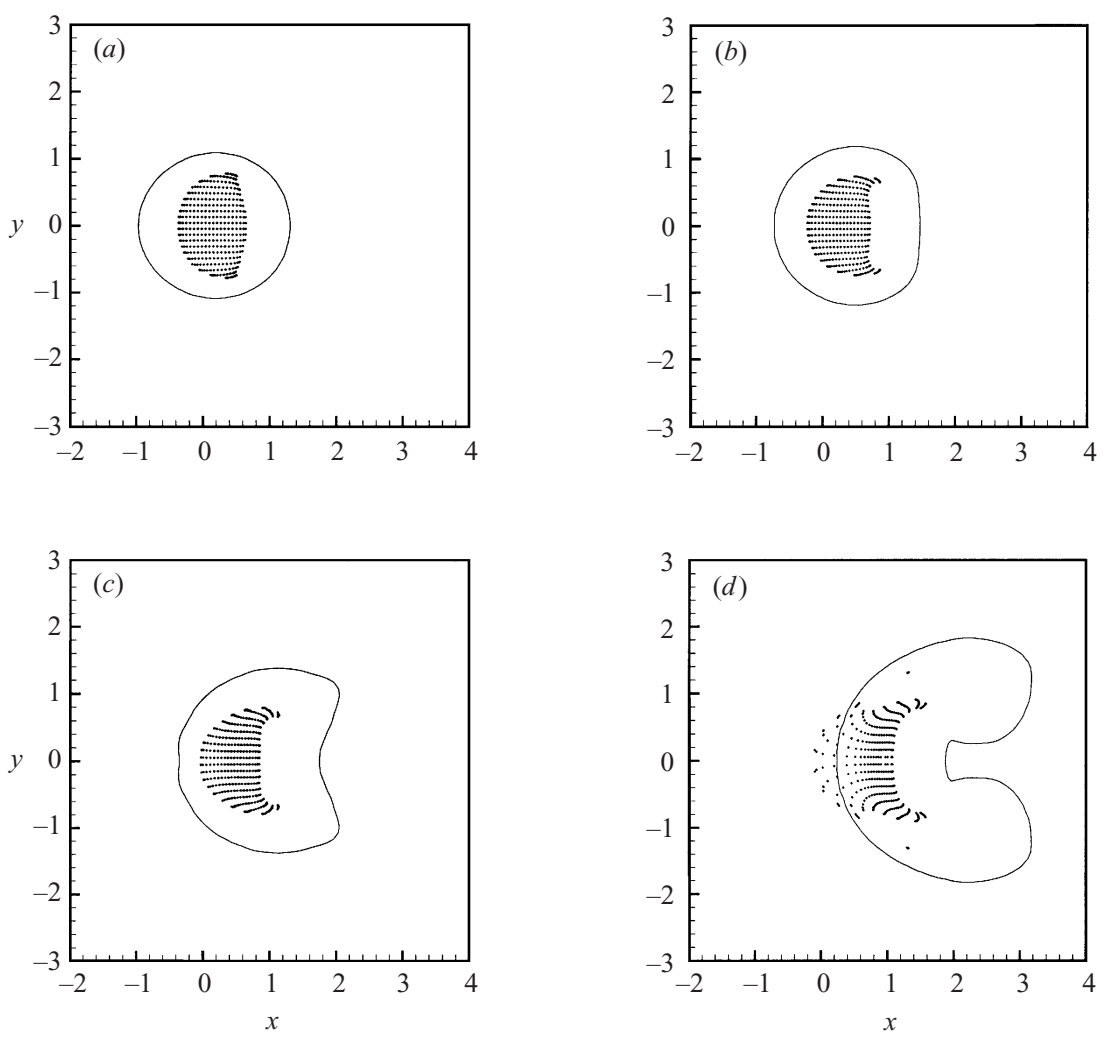

FIGURE 17. As figure 16 but for $U_{\text {jet }} / U_{\infty}=5.4, \delta / D=2.0$, and $\bar{\omega}=3.0$.

17) does not directly translate into increased entrainment of crossflow, at least, very near jet injection. Yet in figure $17(c, d)$, at $z=4$, as the jet emerges from the region of retarded crossflow created by the thicker boundary layer, one sees a relatively rapid deformation of the jet, similar to that in figure $15(c)$ at $z=3$. Hence the mixing characteristics for the thicker boundary layer appear to be similar to those for the thinner boundary layer, just delayed spatially (in the $z$-direction) until the jet emerges from the influence of the boundary layer. This finding is consistent with a slightly higher vortex rollup period $T$ (see table 1) for the thicker boundary layers.

\section{Summary and conclusions}

The near-field evolution of the jet in crossflow has been simulated here using temporally evolving, three-dimensional vortex elements. This simulation allows the examination of the instantaneous flow structure of the jet as well as an ensembleaveraged view of the flow field. Instantaneous images exhibit clear rollup of vortical structures, in addition to tilting and folding of the evolving rings. Folding of the vortex rings is seen, consistent with the ideas of Kelso et al. (1996), to be responsible for the initiation of the counter-rotating vortex pair as well as the stretching and deformation of later-forming vortical structures. Jet penetration as well as periodicity associated with the rollup, folding, and interaction with prior and new vortex ring structures are seen to be dependent on specific flow conditions, including the jet velocity, crossflow velocity, and upstream boundary layer thickness. The influence of these parameters 
on penetration corresponds well with experimental observations (Kamotani \& Greber 1972; Abbitt et al. 1993; Karagozian et al. 1996). If increasing vortex rollup period is seen to suggest increased jet-crossflow interaction and hence potential for entrainment and mixing, then the present results are further consistent with experimental findings (Karagozian et al. 1996). But as noted in $\S 3$, a proper definition of scaled frequency (e.g. a Strouhal frequency) for the transverse jet, as distinct from the free jet, is needed, since the rollup process is influenced by additional parameters in the transverse jet problem.

While instantaneous images provide some evidence for the potential initiation of the CVP through the downstream part of the folded vortex ring structure, ensembleaveraged images (averaged over a single vortex rollup period) provide much stronger evidence of the initiation of a robust CVP. Moreover, the ensemble-averaged velocity field may be used to predict the evolution of streaklines placed upstream of the jet, so that entrainment of crossflow by the jet may be visualized. There is clear evidence of entrainment of crossflow on the lee side of the jet, with a concentration of injectant in the upstream portion of the jet, similar to experimental observations (Kamotani $\&$ Greber 1972). There is also visualization of a fanning of the deflected upstream boundary layer flow about the jet and upward into the lee side of the jet, which suggests origins of the experimentally observed wake vortices within the boundary layer, again consistent with experiments (Fric \& Roshko 1994; Smith \& Mungal 1998).

Entrainment of crossflow into the near-field of the jet is also visualized in these simulations by taking slices of the jet, in the $(x, y)$-plane, at different $z$ locations. Clear evidence of the increasing proportion of crossflow fluid (and the decreasing proportion of injectant) in downstream jet slices is observed. Entrainment of crossflow appears to be clearly enhanced as the jet is deformed and as vortex rollup results in the formation of the CVP. While corresponding enhancement of fluid mechanical mixing as a result of the entrainment cannot be quantified in the present study, since molecular diffusion is not explicitly represented, the conditions for which entrainment increases can be used to identify conditions for which potentially greater mixing of injectant and crossflow can occur.

The authors wish to thank Professors Anthony Leonard, Anatol Roshko, and Frank Marble for several valuable discussions pertaining to this research. This work has been supported by NASA Dryden Flight Research Center under Grant NCC 2-374, by the California Energy Commission/EISG under Grant 51240A/99-01-56, by the University of California Energy Institute, and by NSERC Grant RGPIN217169.

\section{REFERENCES}

Abbitt, J. D. III, Segal, C., McDaniel, J. C., Krauss, R. H. \& Whitehurst, R. B. 1984 Experimental supersonic hydrogen combustion employing staged injection behind a rearward-facing step. J. Prop. Power 9, 472-478.

Andreopoulos, J. \& Rodi, W. 1984 Experimental investigation of jets in a crossflow. J. Fluid Mech. 138, 93-127.

Ashurst, W. T. 1983 Large eddy simulation via vortex dynamics. AIAA Paper 83-1879-CP.

Ashurst, W. T. \& Meiburg, E. 1988 Three-dimensional shear layers via vortex dynamics. J. Fluid Mech. 189, 87-116.

Broadwell, J. E. \& Breidenthal, R. E. 1984 Structure and mixing of a transverse jet in incompressible flow. J. Fluid Mech. 148, 405-412. 
Brzustowski, T. A. 1977 Hydrocarbon turbulent diffusion flame in subsonic cross flow. Turbulent Combustion: Prog. Astro. Aero. 58, 407-430.

Coelho, S. L. V. \& Hunt, J. C. R. 1989 The dynamics of the near field of strong jets in crossflows. J. Fluid Mech. 200, 95-120.

Cottet, G.-H. \& Koumoutsakos, P. D. 2000 Vortex methods-Theory and Practice. Cambridge University Press.

Dahm, W. J. A., Frieler, C. \& TryggVason, G. 1992 Vortex structure and dynamics in the near field of a coaxial jet. J. Fluid Mech. 241, 371-402.

Eroglu, A. \& Breidenthal, R. E. 1991 Effects of periodic disturbances on structure and flame length of a jet in a cross flow. AIAA Paper 91-0317.

FeArn, R. \& Weston, R. 1974 Vorticity associated with a jet in a cross flow. AIAA J. 12, 1666-1671.

FRIC, T. F. 1990 Structure in the near field of the transverse jet. PhD thesis, California Institute of Technology.

Fric, T. F. \& Roshko, A. 1994 Vortical structure in the wake of a transverse jet. J. Fluid Mech. 279, $1-47$.

Gharakhani, A. \& Ghoniem, A. F. 1997 Three-dimensional vortex simulation of time dependent incompressible internal viscous flows. J. Comput. Phys. 134, 75-95.

Hermanson, J. C., Wahba, A. \& Johari, H. 1998 Duty-cycle effects on penetration of fully modulated, turbulent jets in crossflow. AIAA J. 36, 1935-1937.

Hottel, V. O. \& Luce, R. G. 1953 Burning in laminar and turbulent fuel jets. Fourth Symp. (Intl) on Combustion, p. 97. The Combustion Institute.

Johari, H., Pacheco-Tougas, M. \& Hermanson, J. C. 1999 Penetration and mixing of fully modulated turbulent jets in crossflow. AIAA J. 37, 842-850.

Kamotani, Y. \& Greber, I. 1972 Experiments on a turbulent jet in a cross flow. AIAA J. 10, $1425-1429$.

Karagozian, A. R. 1986 a An analytical model for the vorticity associated with a transverse jet. AIAA J. 24, 429-436.

Karagozian, A. R. $1986 b$ The flame structure and vorticity generated by a chemically reacting transverse jet. AIAA J. 24, 1502-1507.

Karagozian, A. R., Wang, K. C., Le, A.-T. \& Smith, O. I. 1996 Transverse gas jet injection behind a rearward-facing step. J. Prop. Power 12, 1129-1136.

Kelso, R. M., Lim, T. T. \& Perry, A. E. 1996 An experimental study of round jets in cross-flow. J. Fluid Mech. 306, 111-144.

Kelso, R. M. \& Smits, A. J. 1995 Horseshoe vortex systems resulting from the interaction between a laminar boundary layer and a transverse jet. Phys. Fluids 7, 153-158.

Krothapalli, A., Lourenco, L. \& Buchlin, J. M. 1990 Separated flow upstream of a jet in a crossflow. AIAA J. 28, 414-420.

Kuzo, D. M. 1995 An experimental study of the turbulent transverse jet. PhD thesis, California Institute of Technology.

LeONARD, A. 1985 Computing three-dimensional incompressible flows with vortex elements. Ann. Rev. Fluid Mech. 17, 523-559.

Lim, T. T., Kelso, R. M. \& Perry, A. E. 1998 A visual study of vortex rings fired transversely into a crossflow. 13th Australian Fluid Mechanics Conference, Monash University, Melbourne, Australia.

Martin, J. E. \& Meiburg, E. 1991 Numerical investigation of three-dimensionally evolving jets subject to axisymmetric and azimuthal perturbations. J. Fluid Mech. 230, 271-318.

Martin, J. E. \& Meiburg, E. 1992 Numerical investigation of three-dimensionally evolving jets under helical perturbations. J. Fluid Mech. 243, 457-487.

Moussa, Z. M., TrischKa, J. W. \& EskinaZI, S. 1977 The near field in the mixing of a round jet with a cross-stream. J. Fluid Mech. 80, 49-80.

Nitsche, M. \& Krasny, R. 1994 A numerical study of a vortex ring formation at the edge of a circular tube. J. Fluid Mech. 276, 139-161.

Parekh, D. E., Leonard, A. \& Reynolds, W. C. 1988 Bifurcating jets at high Reynolds numbers. Rep. TF-35, Thermosciences Division, Department of Mechanical Engineering, Stanford University.

Rudman, M. 1994 Numerical simulation of a jet in crossflow. Intl Colloquium on Jets, Wakes, and Shear Layers, Melbourne, AU. CSIRO. 
SARPKAYA, T. 1989 Computational methods with vortices - The 1988 Freeman Scholar Lecture. Trans. ASME: J. Fluids Engng 111, 5-52.

Schuller, T., King, J., Majamaki, A. \& Karagozian, A. R. 1999 An experimental study of acoustically controlled gas jets in crossflow. Bull. Am. Phys. Soc. 44, 111.

Smith, S. H. \& Mungal, M. G. 1998 Mixing, structure and scaling of the jet in crossflow. J. Fluid Mech. 357, 83-122.

StEWARD, F. R. 1970 rediction of the height of turbulent diffusion buoyant flames. Combust. Sci. Tech. 2, 202-212.

Uenishi, K., Rogers, R. C. \& Northam, G. B. 1989 Numerical predictions of a rearward-facing-step flow in a supersonic combustor. J. Propul. Power 5, 158-164.

Yuan, L. L. \& Street, R. L. 1998 Trajectory and entrainment of a round jet in crossflow. Phys. Fluids 10, 2323-2335.

Yuan, L. L., Street, R. L. \& Ferziger, J. H. 1999 Large-eddy simulations of a round jet in crossflow. J. Fluid Mech. 379, 71-104. 